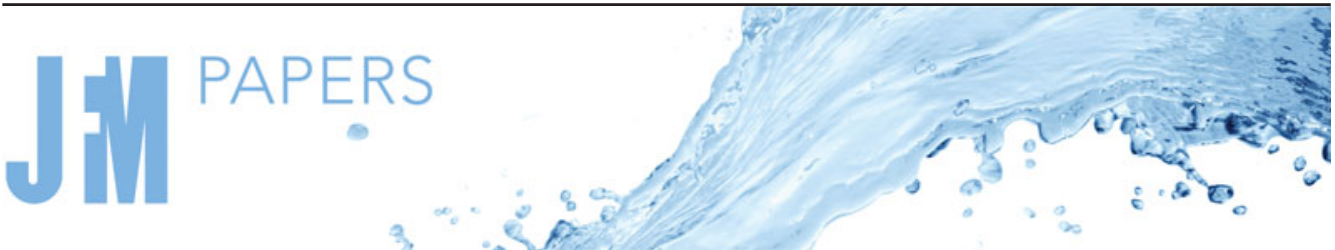

\section{Impact force reduction by consecutive water entry of spheres}

\author{
Rafsan Rabbi ${ }^{1}$, Nathan B. Speirs ${ }^{2}$, Akihito Kiyama ${ }^{1}$, Jesse Belden ${ }^{3}$ \\ and Tadd T. Truscott ${ }^{1, \dagger}$ \\ ${ }^{1}$ Department of Mechanical and Aerospace Engineering, Utah State University, Logan, UT 84322, USA \\ ${ }^{2}$ King Abdullah Univeristy of Science and Technology, Thuwal 23955, KSA \\ ${ }^{3}$ Naval Undersea Warfare Center, Newport, RI 02841, USA
}

(Received 7 July 2020; revised 28 October 2020; accepted 19 December 2020)

Free-falling objects impacting onto water pools experience a very high initial impact force, greatest at the moment when breaking through the free surface. Many have intuitively wondered whether throwing another object in front of an important object (like oneself) before impacting the water surface may reduce this high impact force. Here, we test this idea experimentally by allowing two spheres to consecutively enter the water and measuring the forces on the trailing sphere. We find that the impact acceleration reduction on the trailing sphere depends on the dynamics of the cavity created by the first sphere and the relative timing of the second sphere impact. These combined effects are captured by the non-dimensional 'Matryoshka' number, which classifies the observed phenomena into four major regimes. In three of these regimes, we find that the impact acceleration on the second sphere is reduced by up to $78 \%$ relative to impact on a quiescent water surface. Surprisingly, in one of the regimes the force on the trailing sphere is dramatically increased by more than $400 \%$ in the worst case observed. We explain how the various stages of cavity evolution result in the observed alterations in impact force in this multi-body water entry problem.

Key words: drag reduction, flow-structure interactions

\section{Introduction}

A prevailing myth is that water feels like concrete if one jumps onto it from a great enough height. Although this may seem like an oversimplification, the statement is somewhat truthful. The impact force felt at the time of penetrating a quiescent water surface can be very high (Thompson 1928; Von Karman 1929; Watanabe 1933; Shiffman \& Spencer

$\dagger$ Email address for correspondence: taddtruscott@gmail.com 


\section{R. Rabbi and others}

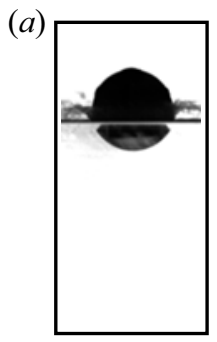

(b)

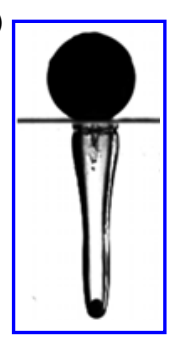

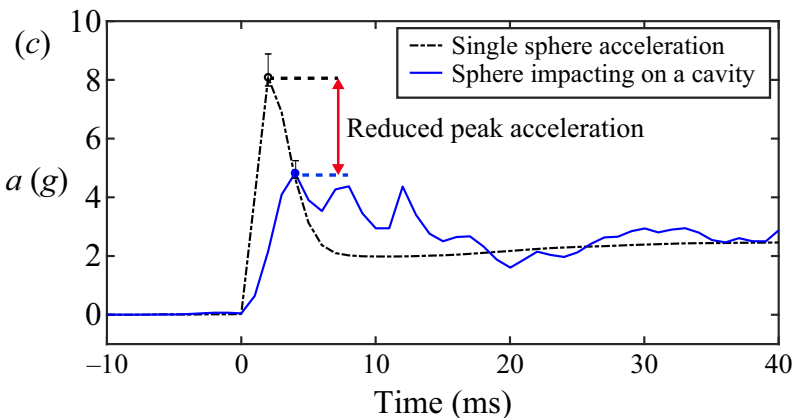

Figure 1. Two different impact phenomena with their impact acceleration data plotted. (a) Free-surface water impact of a $50 \mathrm{~mm}$ Vero plastic sphere $\left(U_{o}=3.76 \mathrm{~m} \mathrm{~s}^{-1}\right)$. (b) The same sphere impacting at the same speed onto a cavity already created by a $10 \mathrm{~mm}$ sphere $\left(3.38 \mathrm{~m} \mathrm{~s}^{-1}\right)$. (c) An accelerometer embedded in the Vero plastic sphere reveals peaks of $\sim 8 g$ for the case in $(a)$ and $\sim 4.8 g$ for the case in $(b)$. The reduction in peak initial impact acceleration between the two indicates that the pre-formed cavity reduces the impact force on the trailing sphere.

1945; May 1975; Grady 1979; Moghisi \& Squire 1981; Korobkin \& Pukhnachov 1988), much higher than the subsequent sustained underwater drag. One such example is shown in figure 1(a), where a $50 \mathrm{~mm}$ sphere dropped from $0.72 \mathrm{~m}$ above the free surface results in an impulse with a peak impact acceleration of $\sim 8 g$ whereas the underwater acceleration is close to a constant value of $\sim 2 g(1 c)$, indicating that at the moment of surface penetration the drag coefficient $C_{d}$ is four times the steady state underwater free fall. This initially high impact force is primarily due to the large rate of change of momentum of the added fluid mass (May 1975; Wang, Lugni \& Faltinsen 2015; Wang, Faltinsen \& Lugni 2019), which is the highest during a submergence depth of $10 \%-20 \%$ of the radius for spheres (figure $1 a$ ) (Shiffman \& Spencer 1945; Moghisi \& Squire 1981). Reducing this peak impact force is of significant interest because it presents structural failure risk to impinging bodies like aircraft landing on water, water landing spacecraft, underwater missiles, divers, base jumpers, etc. (Kornhauser 1964; May 1975; Seddon \& Moatamedi 2006; Guillet et al. 2020). Previous studies have shown that impact forces can be reduced not only through object geometry (McGehee, Hathaway \& Vaughan 1959; Thompson 1965; Li \& Sigimura 1967; May 1970; Qi et al. 2016; Sharker et al. 2019; Güzel \& Korkmaz 2020), but also by modifying the near-surface region via, for example, aeration (Elhimer et al. 2017) or liquid jet-induced acceleration (Speirs et al. 2019a). An interesting extension to the idea of free surface modification is to launch a precursory object to agitate the free surface before entry. Such a concept has been proposed in popular culture (e.g. Mythbusters, Hollywood movies), yet has not received careful scientific investigation.

Here, we present the findings from an experiment investigating the consecutive water entry of two spheres, where the spheres are axially aligned and vertically separated (figure $1 b$ ). Only a few other studies have looked into multiple sphere entry. Yang et al. (2019) studied the water entry of a linear array of magnetic spheres focusing on the cavity shape of the array. Yun, Lyu \& Wei (2019) showed that oblique two sphere entry resulted in contact with the cavity wall and eventual collision of the two spheres, but neither study focuses on the hydrodynamic forces involved. We measure the forces of water entry through an accelerometer embedded in the upper sphere which provides time-resolved measurements of the impact force. The lower sphere hits the water and creates a cavity through which the upper sphere falls, which can result in a reduced impact force on the upper sphere. Figure 1(c) presents an example, where the upper sphere experiences a $\sim 40 \%$ reduction in impact acceleration compared to the case where the same sphere 


\section{Impact force reduction}

impacts the quiescent free surface at the same velocity but without a cavity in front. We propose a modified version of the non-dimensional parameter called the 'Matryoshka' (Mt) number (Hurd et al. 2015; Speirs et al. 2018) based on the cavity characteristics and the vertical spacing between the two spheres, which allows us to build an experimental regime diagram correlating different cavity conditions with the upper sphere impact force reduction and results in the observation of four distinct classes of consecutive two-sphere water entry behaviour.

\section{Experimental methods}

Figure 2(a) illustrates the experimental set-up used for this study. Two spheres of diameter $d_{1}$ and $d_{2}$ are placed on two vertically separated axially aligned platforms held above a glass water tank filled to a height of $1.2 \mathrm{~m}$. The platforms are kept parallel to the water surface with the help of a clamped string and pulley mechanism. When the platforms are released at the same moment, the two spheres free fall and impact the water surface in tandem. The lower sphere $\left(d_{1}\right)$ impacts the water surface first, creating a cavity. The trailing upper sphere impacts on different stages of the cavity in front depending on the spacing between the two spheres, and the time difference of impact $\Delta t$. The impact acceleration for the upper sphere is recorded by an accelerometer embedded in an inertial measurement unit (IMU) placed inside the upper sphere. The impact events are captured with a Photron SA-3 high-speed camera at 1000 f.p.s. The videos taken were used to calculate different important parameters such as the cavity opening diameter, pinch-off time and to differentiate between different modes of consecutive two-sphere entry. The spheres are kept at heights of $h_{2}$ and $h_{1}$ from the water surface, the spacing $\left(\Delta h=\left(h_{2}-h_{1}\right)\right)$ between the spheres varies from 0.07 to $1.24 \mathrm{~m}$. For every two-sphere impact combination tried, a quiescent upper sphere drop from the same drop height $h_{2}$ was also done to measure the peak quiescent impact acceleration for comparison with the two-sphere peak impact acceleration.

The upper sphere is a 3D-printed Vero plastic sphere with a fixed diameter of $d_{2}=$ $50 \mathrm{~mm}$. The sphere consists of two separable parts for helping in removing the IMU between experiments. The two parts of the sphere are pressed together with the help of butyl-ethylene tape to seal off and prevent water from entering. The contact area of the two parts is located one third the diameter from the top of the sphere, minimizing its influence on water entry events. Weights are inserted in the upper sphere to make it bottom heavy resulting in a density of $2290 \mathrm{~kg} \mathrm{~m}^{-3}$ for the sphere as a whole. Five different diameter steel spheres $\left(d_{1}=10-38 \mathrm{~mm}\right.$, density $\left.7800 \mathrm{~kg} \mathrm{~m}^{-3}\right)$ are used as the lower sphere, sprayed with Cytonix WX-2100 coating to make them hydrophobic, resulting in a surface contact angle of $117^{\circ}$ and increased the roughness of the spheres to $R_{z}=50.2 \pm 21.4 \mu \mathrm{m}(95 \%$ confidence). The Vero plastic upper sphere has a hydrophilic surface of wetting angle $\theta=80 \pm 8^{\circ}$ and surface roughness $R_{z}=7.2 \pm 1.2 \mu \mathrm{m}$ (95\% confidence) such that it forms a cavity during water entry (see Table 1 in Appendix for more information).

The upper sphere housed an IMU built in house, with two three-axis accelerometers, one gyroscope and one magnetometer embedded. The two accelerometers on-board were one low range and one high range. The low range accelerometer has a measurement range of $\pm 16 \mathrm{~g}$, it is a MPU-9250 motion tracking device manufactured by Invensen Inc. The high range accelerometer is a chip called H3LIS331DL produced by STMicroelectronics, and was set to a maximum range of $\pm 100 \mathrm{~g}$. Both would register data for any drop event, whenever possible the data from the low accelerometer are reported, because the high accelerometer is more prone to noise. Data from both are comparable for cases where acceleration values were within $\pm 16 \mathrm{~g}$. The accelerometer sampling rate is limited to 


\section{R. Rabbi and others}
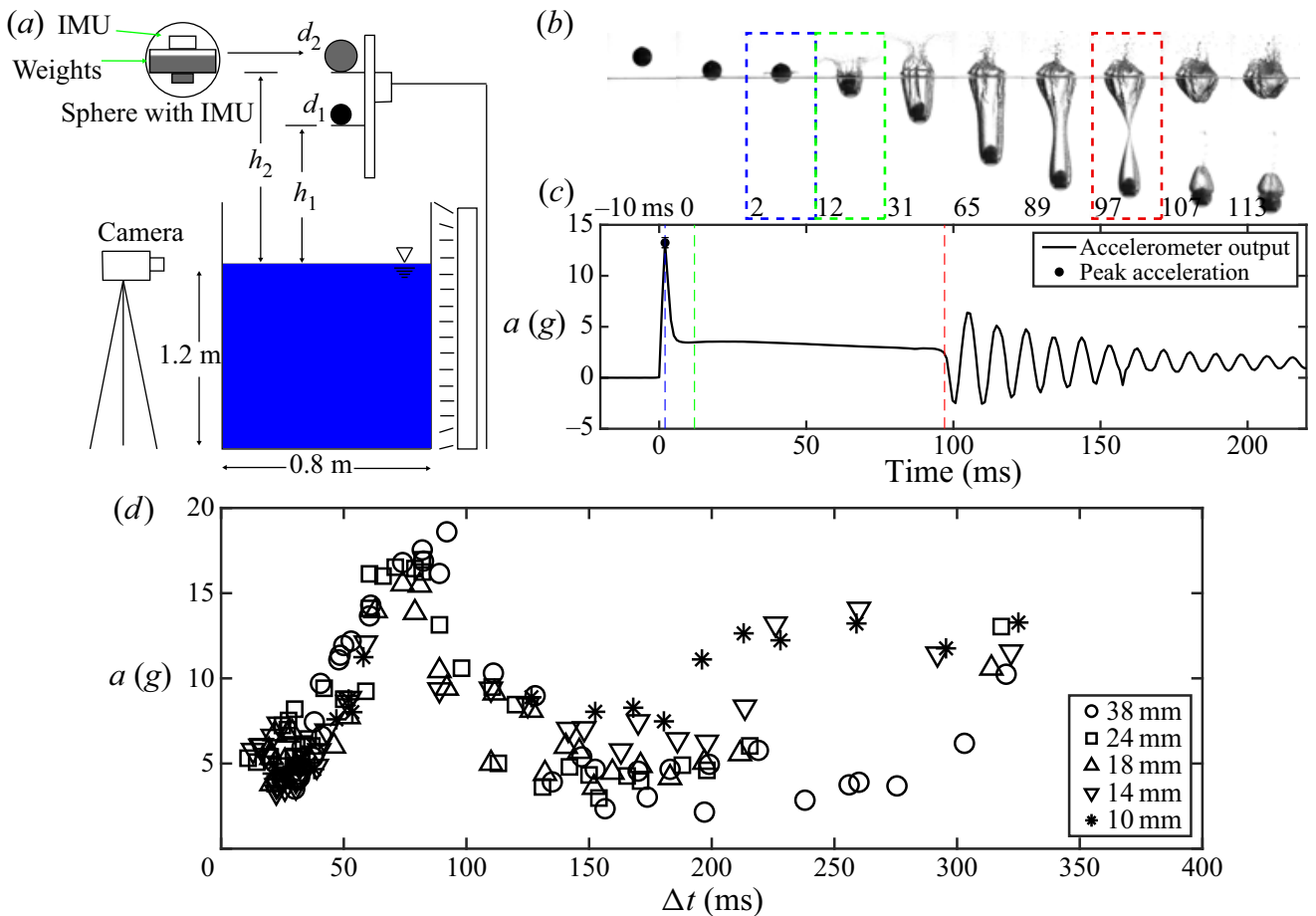

Figure 2. A schematic diagram of the experimental set-up for consecutive two-sphere water entry is shown in (a). The spacing $\left(h_{2}-h_{1}\right)=\Delta h$ is varied to attain different modes of two-sphere impact. (b) A quiescent drop where the upper sphere impacts the undisturbed water pool from height $h_{2}=1.27 \mathrm{~m}$ and creates a cavity. (c) The data taken by the accelerometer inside the sphere show the acceleration at different stages of the quiescent sphere impact and entry, with the blue, green and red coloured dashed boxes $(b)$ and lines $(c)$ indicating the time of peak acceleration, steady state underwater drag and starting point of cavity pinch-off. $(d)$ The measured peak impact accelerations experienced by the upper sphere for the consecutive two-sphere impacts studied herein, presented with respect to the variation of the time difference $\Delta t$ between impacts. Variation in $\Delta h$ results in different $\Delta t$. Different markers represent the different diameter $\left(d_{1}\right)$ lower spheres (that travel in front of the upper sphere and impact the quiescent water pool first) used in the study.

$1000 \mathrm{~Hz}$. The root sum square of the acceleration values from the three axes is calculated and reported as the total acceleration.

Figure 2(b) shows a typical quiescent upper sphere impact event, with the acceleration output from the IMU shown in figure 2(c). The sphere impacts the free surface at $0 \mathrm{~ms}$, and a sudden increment of acceleration is registered. The peak acceleration is reached soon after, shown by the black dot and the time marked by the blue dashed line. This impact pulse lasts fleetingly until $\sim 8 \mathrm{~ms}$, after which the sphere travels downwards with an air cavity in its wake until $97 \mathrm{~ms}$, when the cavity pinches-off and divides into two halves. The sphere travels downwards after pinch-off with an air bubble attached to it. The bubble experiences pressure perturbations, which are registered in the accelerometer as oscillations after $97 \mathrm{~ms}$ (red dashed box and line in figure $2 b, c$ ). The quiescent peak values are compared with the peak accelerations measured from consecutive two-sphere impact drops (figure $2 d$ ) to calculate the change in impact acceleration.

\subsection{Uncertainty}

The uncertainty relating to the measurements are calculated and the uncertainty bands in the figures represent $95 \%$ confidence interval of the measurement (Coleman \& 


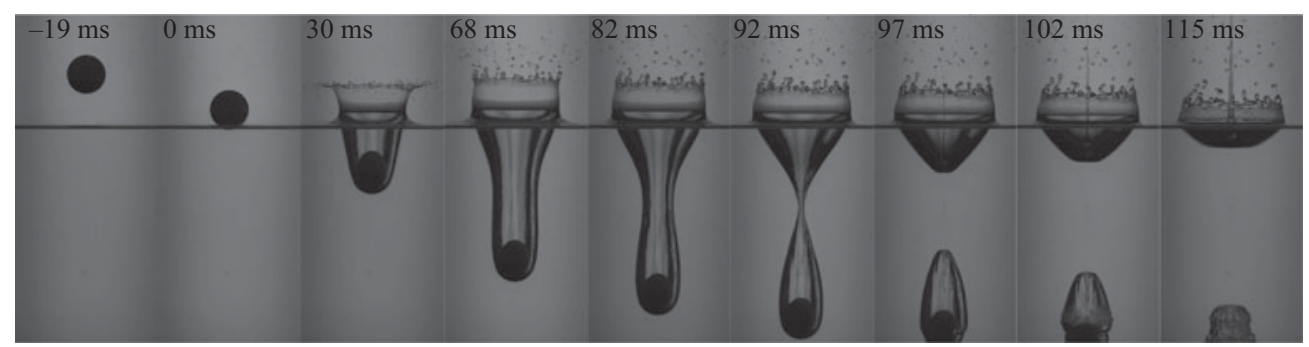

Figure 3. A typical lower sphere impact on quiescent water surface without a trailing upper sphere. A $38 \mathrm{~mm}$ hydrophobic steel sphere impacts a quiescent water pool $\left(U_{o} \sim 3.2 \mathrm{~m} \mathrm{~s}^{-1}\right)$ creating a subsurface air cavity (0-68 ms). The cavity elongates in time with the downwards moving sphere (30-92 ms) and finally collapses on itself in a deep-seal (92 ms) pinch-off. After the deep seal, the cavity is divided in two parts, a cavity bubble attached with the sphere moving downwards $(97 \mathrm{~ms})$ and the upper bowl shaped distortion of the free surface, which eventually create a Worthington jet (97-115 ms).

Steele 2018). The method of calculating uncertainties for peak acceleration values and non-dimensional 'Matryoshka' number is explained in detail in appendix A.1. The uncertainty bands are placed on acceleration plots whenever possible.

\section{Results and discussion}

\subsection{Scaling analysis and formulation of 'Matryoshka' number}

When an object impacts a water pool it displaces some of the water with air and accelerates fluid downwards as the object falls through the pool, leaving an air filled cavity in its wake (Truscott, Epps \& Belden 2014). Creating a cavity in front of an impacting object can be conducive to reducing its impact acceleration, as evident from figure 1 $(c)$. The state of the cavity over time indicates the local liquid flow field surrounding the cavity (Truscott, Epps \& Techet 2012; Mansoor et al. 2014), which may help explain the change in impact acceleration for any trailing object. Thus, understanding cavity creation and evolution is paramount for determining why and how an air cavity may reduce impact force.

Objects with rough and hydrophobic surfaces almost always create cavities even at very low impact velocities (Duez et al. 2007; Zhao, Chen \& Wang 2014; Speirs et al. 2019b). Figure 3 shows such a case where a $38 \mathrm{~mm}$ hydrophobic sphere creates an axisymmetric cavity at an impact velocity of $\sim 3.2 \mathrm{~m} \mathrm{~s}^{-1}$. The cavity elongates with the downward moving sphere, until the point when hydrostatic pressure forces the cavity to seal near the cavity mid-point at $92 \mathrm{~ms}$. This sealing event is popularly referred to as 'deep-seal' pinch-off. After pinch-off the cavity divides into two parts, a pulsating air bubble attached to the downwards moving sphere and the upper bowl shaped distortion in the free surface retreating upwards creating a high-speed axisymmetric 'Worthington' jet (Worthington \& Cole 1897; Gekle \& Gordillo 2010), as seen in figure 2(a) from 92 to $115 \mathrm{~ms}$. Different impact velocities and sphere sizes result in different cavity behaviours which can be classified by cavity seal type (Aristoff \& Bush 2009; Speirs et al. 2019b). For example, the cavity shown in figure 3 is referred to as deep-seal cavity because of the characteristic mid depth deep-seal pinch-off.

Increasing the impact velocity for the same sphere sizes results in surface seal (Aristoff \& Bush 2009; Mansoor et al. 2014; Speirs et al. 2019b), which is distinguished by the splash crown sealing above the free surface, and the resulting detachment and pull away of the cavity below the free surface. In the context of consecutive two-sphere water entry, one might expect the pinch-off (or seal) event from the cavity of the first sphere to affect 


\section{R. Rabbi and others}

the dynamics of the trailing sphere. For two axially aligned, vertically separated spheres (upper sphere diameter $d_{2}$, lower sphere diameter $d_{1}$ ) as shown in figure 2(a), varying the spacing $\Delta h=\left(h_{2}-h_{1}\right)$ between the two spheres will result in the upper sphere interacting with the cavity either before or after pinch-off, which we anticipate will lead to different sphere-cavity interaction modes. Taking the pinch-off time as a characteristic time scale, we propose to characterize consecutive two-sphere water entry with a modification of the non-dimensional parameter known as the 'Matryoshka' number $M t$. This term has been used in prior research to describe successive cavity formation from multi-droplet impacts using droplet frequency and cavity formation time as the fundamental time scales (Hurd et al. 2015; Speirs et al. 2018).In a physical sense, $M t$ can be considered a ratio of the time to completion of a single event to the consecutive initiation of the same event by the second sphere. Here, we define

$$
M t=\frac{\Delta t}{t_{p}} .
$$

where $\Delta t=\left|t_{2}-t_{1}\right|$ is the time difference between the two spheres passing the free surface (figure $4 a$ ), and $t_{p}$ is the pinch-off time of the first cavity. Thus, $M t$ parameterizes the state of the cavity formed by the first sphere at the time when the second sphere interacts with it. The case $M t<1$ indicates that the first cavity has not gone through pinch-off, which results in cases where the upper sphere interacts with an elongating cavity. The cavity opening diameter $d_{c}$ (itself a function of time and the lower sphere diameter Duclaux et al. 2007; Aristoff \& Bush 2009) is measured at time $t_{2}$. If $d_{c}<d_{2}$, the upper sphere falls on the cavity when impacting the water pool, and we name these 'on cavity' cases (figure $4 b$ ). When $d_{c}>d_{2}$, then the upper sphere falls through the cavity opening, which we call the 'inside cavity' case, with $d_{c} / d_{2}=1$ working as the transition between the two cavity cases predicted for $M t<1$. For $M t>1$, the trailing sphere interacts with the upper detached portion of the cavity, either falling through $\left(d_{c}>d_{2}\right)$ or falling on $\left(d_{c}<d_{2}\right)$ the bowl shaped retreating free surface; or falling through a Worthington jet resulting from cavity pinch-off at higher $M t$ (figure $4 b$ ). We call these cases 'bowl' and 'on jet', respectively. Figure 5(a) shows snapshots of these predicted modes of consecutive two-sphere water entry from actual experiments, with the modes observed at various $M t$. The experimental map of the consecutive two-sphere entry cases tested herein (figure $5 b$ ) illustrates that the ratio $d_{c} / d_{2}=1$ does work as a separation criterion between the on cavity and inside cavity cases. Note that figure 5(b) also denotes the existence of two different on bowl modes (on cavity bowl and inside cavity bowl as expected from figure $4 b$ ). These two can also mostly be separated using the $d_{c} / d_{2}=1$ condition. The transition from on cavity to bowl mode happens at $M t=1$, as predicted, denoted by the magenta dashed line in figure 5(a), but the transition from inside cavity to inside bowl cases happens earlier $(M t=0.65$, vertical black dashed line on figure $5 b)$. This discrepancy in transition $M t$ between the two pre-pinch-off cavity modes is surprising, but can be explained analytically, as shown in later discussion in $\S 3.2$.

To determine $M t$ as formulated in (3.1) requires experimental measurements of $\Delta t$ and $t_{p}$. To make our results more broadly useful to other researchers, we seek to redefine $M t$ in terms of independent variables. Prior research has shown that the deep-seal pinch-off time can be written as $t_{p}=\beta \sqrt{d_{s} /(2 g)}$, (Glasheen \& McMahon 1996; Duclaux et al. 2007; Truscott \& Techet 2009), where $\beta$ is an experimental constant with different values ranging from 1.72 to 2.285 proposed in the literature (Duclaux et al. 2007; Bergmann et al. 2009; Marston, Vakarelski \& Thoroddsen 2012). This equation works well for low Froude number $(F r)$ impacts, where $F r=U_{o}^{2} /\left(g d_{s}\right), U_{o}$ is the impact velocity, $d_{s}$ is the 
(a)

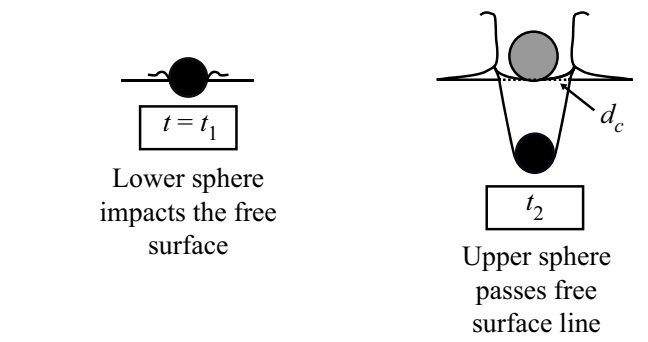

Definition of Matryoshka number:

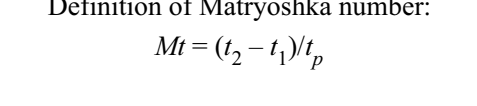
$M t=\left(t_{2}-t_{1}\right) / t_{p}$

(b)
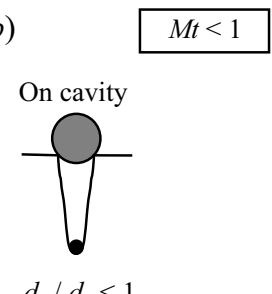

$$
d_{c} / d_{2}<1
$$
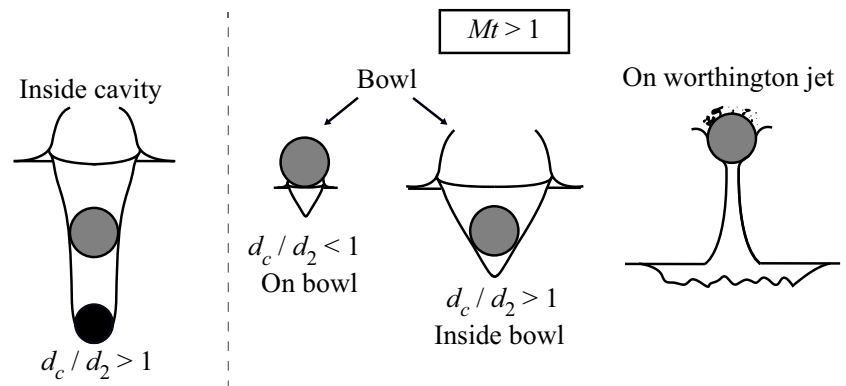

Figure 4. The formulation of the non-dimensional 'Matryoshka' number and predicting different modes of two-sphere water entry using cavity pinch-off time information. (a) When two spheres vertically separated by a distance $\Delta h$ are released from their rest position as shown in figure $2(a)$, the variation in spacing will result in the upper sphere impacting various stages of the cavity created by the lower sphere. Taking the characteristic deep-seal pinch-off time as $t_{p}$, a non-dimensional time parameter called 'Matryoshka' number can be defined. (b) The case $M t=1$ predicts the boundary between different modes of consecutive two-sphere impact. For $M t<1$, the upper sphere can interact with a still growing cavity, yielding pre-pinch-off cavity cases: 'on cavity' and 'inside cavity'. Beyond $M t>1$, the upper sphere should interact with the collapsed cavity, resulting either in two different 'bowl' modes based on the cavity opening diameter $d_{c}$, or with the Worthington jet ('on jet' cases).

sphere diameter and $g$ is the gravitational constant. In this study, experimentally calculated $\beta=2.03 \pm 0.0974$ (95\% confidence) is used (figure $5 c$ ). For higher $F r$ where surface seals are expected to happen, this equation overestimates the pinch-off time marginally (figure 16a), but the lack of a good consensus in the literature about the surface seal time and the scale of the experiments carried out in this paper $(F r<400)$ makes this equation a good approximation. The time spacing $\Delta t$ can be calculated in terms of sphere drop heights using the free-fall relationship between the two spheres. If the upper sphere takes time $\Delta t=\left(t_{2}-t_{1}\right)$ to pass the free-surface line, then $\Delta t=\left(\sqrt{2\left(h_{1}+\Delta h\right) / g}-\sqrt{2\left(h_{1}\right) / g}\right)$. Substituting this in (3.1) and using the pinch-off time relation $t_{p}=\beta \sqrt{d_{1} / 2 g}$ yields,

$$
M t=\frac{2\left(-\sqrt{h_{1}}+\sqrt{h_{1}+\Delta h}\right)}{\beta \sqrt{d_{1}}},
$$

which is as an alternative interpretation for the definition of the $M t$ number. Not only is (3.2) easier to use for prediction than (3.1), but it also explains how the lower sphere diameter and the height difference between the two spheres are required to adequately explain the phenomenon (figure 16b). 


\section{R. Rabbi and others}

(a)

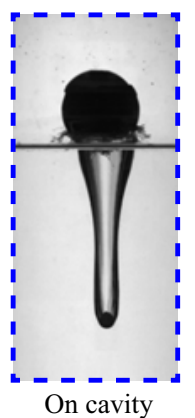

(b)

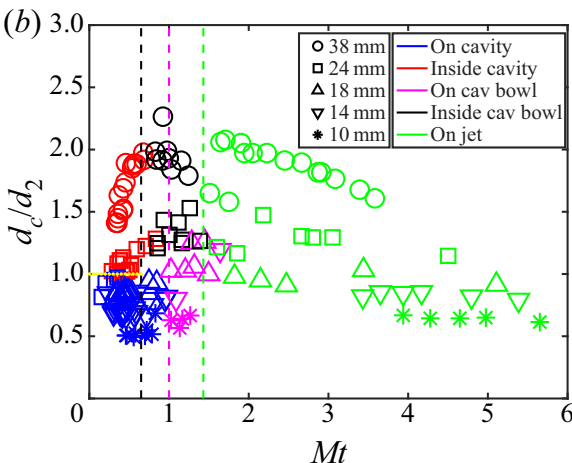

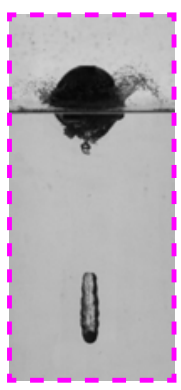

On bowl
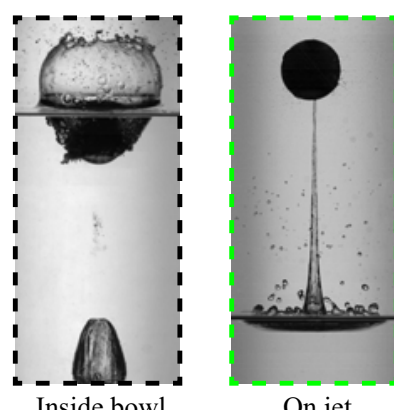

On jet

(c)

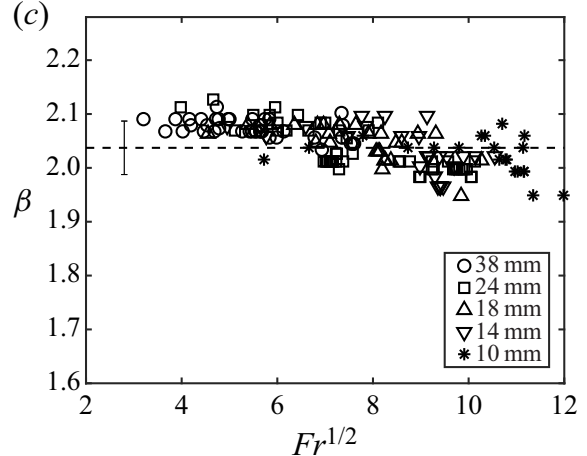

Figure 5. (a) Snapshots of five different two-sphere entry modes observed through experiments. (b) The experimental map of $M t$ and ratio of cavity to the upper sphere diameter $d_{c} / d_{2}$. The different coloured markers denote different two-sphere entry modes, with red, blue and green denoting inside cavity, on cavity and on jet cases; black and magenta represent cases belonging to the two different bowl modes: inside cavity bowl and on cavity bowl, respectively. The dotted yellow line at $d_{c} / d_{2}=1$ marks the separation between the inside cavity and on cavity cases, as shown in figure $4(b)$. The transitions between the modes are represented by the vertical dashed black line at $M t=0.65$ between pre-pinch-off inside cavity and post-pinch-off inside bowl modes, the vertical dashed magenta line at $M t=1$ between on cavity and on cavity bowl cases, and the vertical dashed green line at $M t=1.43$ between post-pinch-off bowl and on jet modes. The emergence of these values as separation criteria is explained in $\S 3.2$. (c) The experimental value of $\beta$ for different sphere diameters over the Froude $(F r)$ number range tried herein for 169 different single-sphere drop experiments. The black dashed line shows the averaged value of $\beta$ to be $2.037 \pm 0.097$ (95\% confidence with vertical error bar).

\subsection{Consecutive sphere entry: different modes}

As explained in the previous section, $M t<1$ results in pre-pinch-off cavity cases (on cavity and inside cavity) depending on the ratio $d_{c} / d_{2}$ (figures $\left.4(b), 5(b)\right)$, and $M t>1$ indicates the post-pinch-off cases (bowl and on jet). Figures 6 and 7 present time-series image sequences of all two-sphere modes including two different bowl cases (figure $7 a, b$ ), with their dynamic acceleration response plotted with the image sequences (figures $6(c)$, $7(d))$. The accelerations at impact are reduced for both on cavity and inside cavity cases compared to the quiescent case peak. In the bowl cases, the initial impact pulse has a higher peak than the quiescent case, indicating a higher impact force experienced during free-surface entry. For the on jet case, the peak acceleration value is significantly smaller than the quiescent peak. The time of the peak accelerations for the two-sphere cases happen later than the quiescent case (see figures $6(a-c), 7(a-d)$ ), since the sphere interacts with modified free-surface conditions.

Figure 8 shows a regime diagram where reduction in acceleration for varying $M t$ is plotted for the range of experimental conditions. Reduction in acceleration is computed as 


\section{Impact force reduction}

(a)

(b)

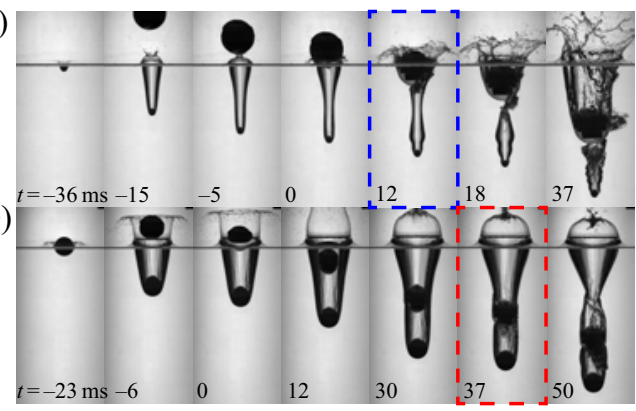

(c)

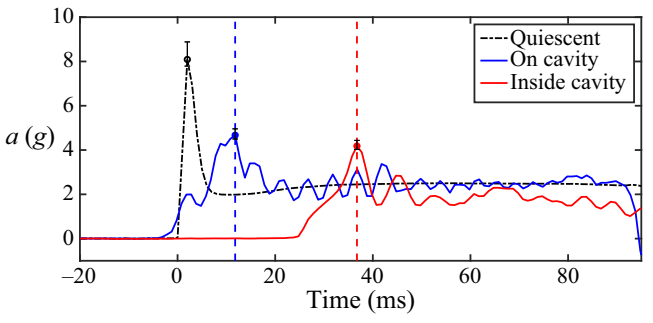

Figure 6. Time series for two example pre-pinch-off cavity cases in consecutive sphere entry. A hydrophobic sphere $\left(d_{1}=10 \mathrm{~mm}(a)\right.$ and $\left.38 \mathrm{~mm}(b)\right)$ impacts the quiescent free surface and creates a cavity, followed by a $50 \mathrm{~mm}$ sphere with embedded IMU in free fall from a height of $h_{2}=0.72 \mathrm{~m}$, where $\Delta h=0.14$ and 0.11 respectively for $(a)$ and $(b)$. Acceleration of both cases is shown in $(c)$. The time the upper sphere passes the free-surface line is considered as $t=0 \mathrm{~ms}$ for each of the cases, yielding $M t \sim 0.82$ and $\sim 0.35$ for the on cavity $(a)$ and the inside cavity $(b)$ cases, respectively. The blue and red dashed boxes and lines in $(a-c)$ denote the time of peak acceleration felt by the upper sphere for on cavity and inside cavity cases. The peak acceleration in both cases are significantly less than the quiescent value of $8 \mathrm{~g}$. The uncertainty bands are as shown. See supplemental movies S1 and S2 available at https://doi.org/10.1017/jfm.2020.1165.

$(a)$

(b)

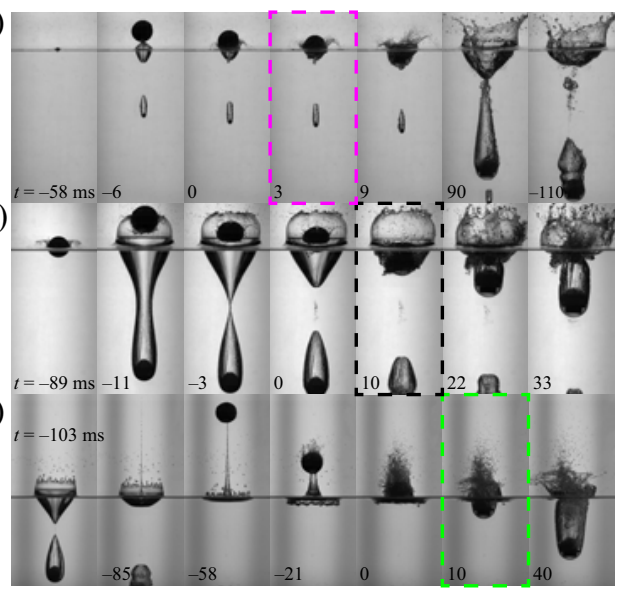

(d)

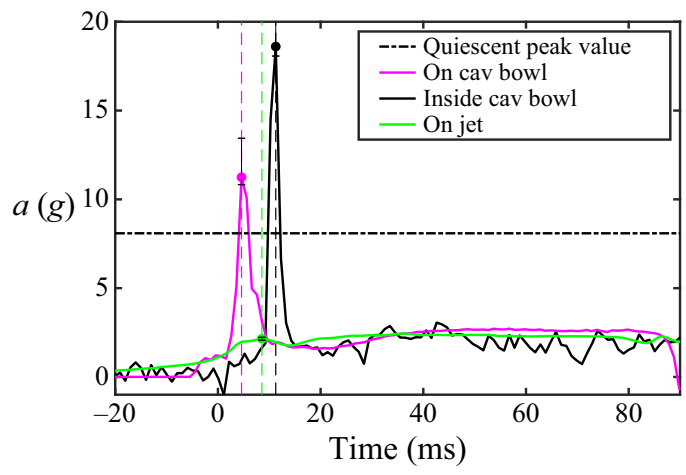

Figure 7. A hydrophobic sphere $\left(d_{1}=10 \mathrm{~mm}(a)\right.$ and $\left.38 \mathrm{~mm}(b, c)\right)$ impacts the quiescent free surface and creates a cavity, followed by the $50 \mathrm{~mm}$ upper sphere in free fall from a height of $h_{2}=0.72 \mathrm{~m}$, where the cases are: (a) inside cavity bowl is $\Delta h=0.22$ and $M t \sim 1.26,(b)$ on cavity bowl is $\Delta h=0.3$ and $M t \sim$ 1.05 and $(c)$ on jet is $\Delta h=0.54$ and $M t \sim 2.20$. (d) Acceleration of each of the cases in $(a-c)$ with peak acceleration time for all marked with magenta, black and green dashed boxes $(a-c)$ and lines $(d)$. The black dashed-dotted horizontal line at $a \sim 8 g$ shows the peak acceleration value for the quiescent case where the upper sphere dropped from the same height $(0.72 \mathrm{~m})$ impacts a quiescent free surface without a cavity in front of it (figure $6 c$ ). The bowl cases show significant increase in acceleration than the quiescent value of $8 \mathrm{~g}$, but the on jet case shows dramatic reduction in peak impact acceleration, which never rises beyond the level of free-fall acceleration through water. See supplemental movies S3-S5.

$1-a / a_{q}$, where $a$ is the measured peak acceleration of the trailing sphere in a two-sphere water entry, and $a_{q}$ is the peak acceleration of the same sphere impacting quiescent water from the same drop height $\left(h_{2}\right)$. Similar to the standalone cases presented in figures 6 and 7 , on cavity $(0<M t<1)$ and inside cavity cases $(0<M t<0.65)$ experience a notable reduction in impact acceleration, with a downward linear trend in reduction values present for both as $M t$ increases. Alternatively, the bowl cases experience an increase in impact 


\section{R. Rabbi and others}

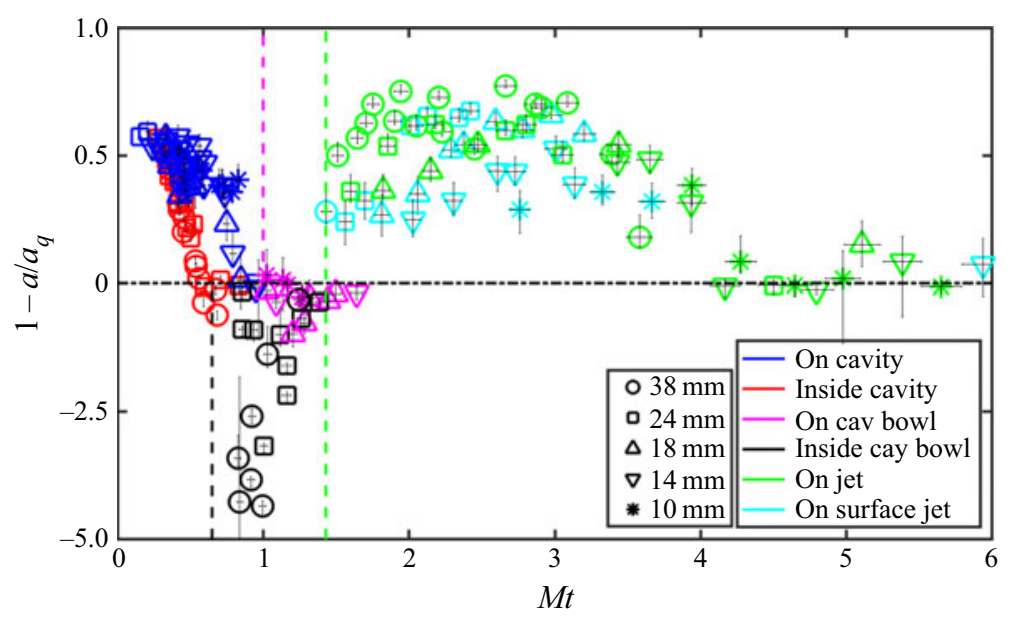

Figure 8. Percentage reduction in the initial impact force for the upper sphere versus $M t$ for different two-sphere water entry modes. Here, $a$ is the peak impact acceleration for the second of the two-sphere impact cases, and $a_{q}$ is the corresponding quiescent impact peak acceleration of the same sphere dropped from the same height. Cavity impact types are the same as figure 4. Significant force reductions are achieved in the inside cavity $(0<M t<0.65)$, on cavity $(0<M t<1)$ and on jet $(1.43<M t<4)$ cases. The transition from inside cavity to on bowl occurs at $M t \sim 0.65$ (black dashed line) and on cavity to on bowl mode at $M t \sim 1$ (magenta dashed line), with both bowl modes showing a significant increase in impact acceleration. The inside cavity bowl and on cavity bowl cases transition to on jet cases at $M t \sim 1.43$ indicated by the dashed vertical green line. A variation of the on jet cases occurs when the splash domeover at the free surface suppresses the Worthington jet, creating a small jet-like water column at the surface we term the 'on surface jet', marked with cyan coloured markers. The horizontal black dashed-dot line at $1-a / a_{q}=0$ in the $y$-axis indicates $a=a_{q}$, while the vertical green dashed line represents the experimentally found transition from both on bowl modes to both on jet modes. Uncertainty bands are marked with $95 \%$ confidence with more information available in appendix A.1. Note: the $y$-axis values above and below 0 are scaled differently.

acceleration, evident from the negative reduction in acceleration in figure 8 up to $M t \sim$ 1.43. The on jet cases experience dramatic reduction in impact acceleration in $1.43<$ $M t<4$ range, with the mode extending to $M t \sim 6$.

We can gain a mechanistic understanding of how the peak accelerations are reduced for some regimes (on cavity, inside cavity, on jet) and dramatically increased for others (on bowl, inside bowl) by considering the contributions to the large force during the first few moments of impact. For inertially dominated regimes, this peak force stems from the rate of change of momentum of the added fluid mass (Shiffman \& Spencer 1945; May 1975). For impact of a sphere on a quiescent free surface, Shiffman \& Spencer (1945) derived an expression for this force invoking conservation of momentum during water impact such that $M U_{o}=(M+m) U$, where $M$ is the sphere mass, $m$ is the added mass of the fluid, $U_{o}$ the impact velocity and $U$ the instantaneous velocity of the sphere and added fluid mass after impact. Defining $b=s / R$, where $R$ is the sphere radius and $s$ the submergence below the initial free-surface plane, the vertical force on the sphere is given as

$$
F_{z}=-M \frac{\mathrm{d} U}{\mathrm{~d} t}=\frac{U_{o}^{2}}{R} \frac{1}{\left(1+\frac{m}{M}\right)^{3}} \frac{\mathrm{d} m}{\mathrm{~d} b}
$$




\section{Impact force reduction}

(see appendix A.2 for detailed derivation). Using the typical definition of the vertical force $F_{z}=\rho \pi R^{2} U_{o}^{2} C_{d} / 2$, where $C_{d}$ is the impact drag coefficient, we get,

$$
C_{d}=\frac{2}{\rho \pi R^{3}} \frac{1}{\left(1+\frac{m}{M}\right)^{3}} \frac{\mathrm{d} m}{\mathrm{~d} b},
$$

which Shiffman \& Spencer (1945) report for values of $m / M$. Thus, the vertical force during early water entry has a first-order dependence on the rate of change of added mass with submergence, $\mathrm{d} m / \mathrm{d} b$. In the on cavity and inside cavity cases, the leading sphere opens a hole on the surface such that the first contact between the water and the sphere is below the free surface and at some higher azimuthal angle on the sphere (see schematic in figure 9). In the quiescent sphere impact, the value of $\mathrm{d} m / \mathrm{d} b$ peaks at small submergence of $b \approx 0.1$ (Shiffman \& Spencer 1945; May 1975). Therefore, one might expect that for on cavity or inside cavity cases for which the bottom of the sphere does not initially contact the water, the value of $\mathrm{d} m / \mathrm{d} b$ and thus the impact force would be smaller than in the quiescent case, as is observed. While the on cavity or inside cavity cases occur prior to first cavity pinch-off, for the on bowl and inside bowl cases the cavity has pinched off and the fluid has significant upward momentum as the bowl retracts. We can rationalize the effect of this to first order by again considering conservation of momentum before and after impact $M U_{o}-$ $m U_{m}=(M+m) U$, where $U_{m}$ is the average velocity of the added fluid mass just before impact (assumed to be in the direction opposite of $U_{o}$ ). Following the same derivation for impact on a quiescent free surface from Shiffman \& Spencer (1945), we arrive at the expression,

$$
F_{z}=\frac{\left(U_{o}-\frac{m}{M} U_{m}\right)}{\left(1+\frac{m}{M}\right)^{3}} \frac{\left(U_{o}+U_{m}\right)}{R} \frac{\mathrm{d} m}{\mathrm{~d} b}
$$

(see derivation in appendix A.2). Now, the vertical force depends not only on $\mathrm{d} m / \mathrm{d} b$, but also increases with $U_{m}$ assuming $m / M$ is sufficiently small. Thus, one would expect the force to increase relative to the quiescent case due to the upward momentum of the retracting bowl that must be reversed during impact. Furthermore, in most on bowl and inside bowl cases, the shape of the surface at the moment of water impact is contoured to the sphere rather than flat (see schematic in figure 9). Therefore, the rate of change of added mass with submergence, $\mathrm{d} m / \mathrm{d} b$, would be larger than impact of a sphere with a flat free surface. Intuitively, one can think of a disk impacting a flat free surface, for which $\mathrm{d} m / \mathrm{d} b$ would go to infinity if the surface were not first deformed by an over-pressurized air layer (May 1975). These two effects - the upward momentum of the fluid prior to impact, and larger rate of change of added mass during impact - combine to make the impact force larger for on bowl and inside bowl cases relative to the quiescent case.

While the trends predicted by this first-order analysis explain the observations of force increase for the bowl cases and force reduction for on and inside cavity cases, the experimental data show a wide range in the peak acceleration in these regimes (see figure 8). The impact acceleration increases with increasing $M t$ for inside cavity cases, which can be understood qualitatively by considering the cavity evolution relative to the second sphere impact. For small $M t$, the cavity is open and cylinder-like at the moment of impact, resulting in water contact near the equator of the sphere where $\mathrm{d} m / \mathrm{d} b$ is small. As $M t$ increases, sphere impact happens closer to the time of pinch-off and the cavity is more conical, resulting in sphere wetting closer to the bottom of the sphere where $\mathrm{d} m / \mathrm{d} b$ 


\section{R. Rabbi and others}

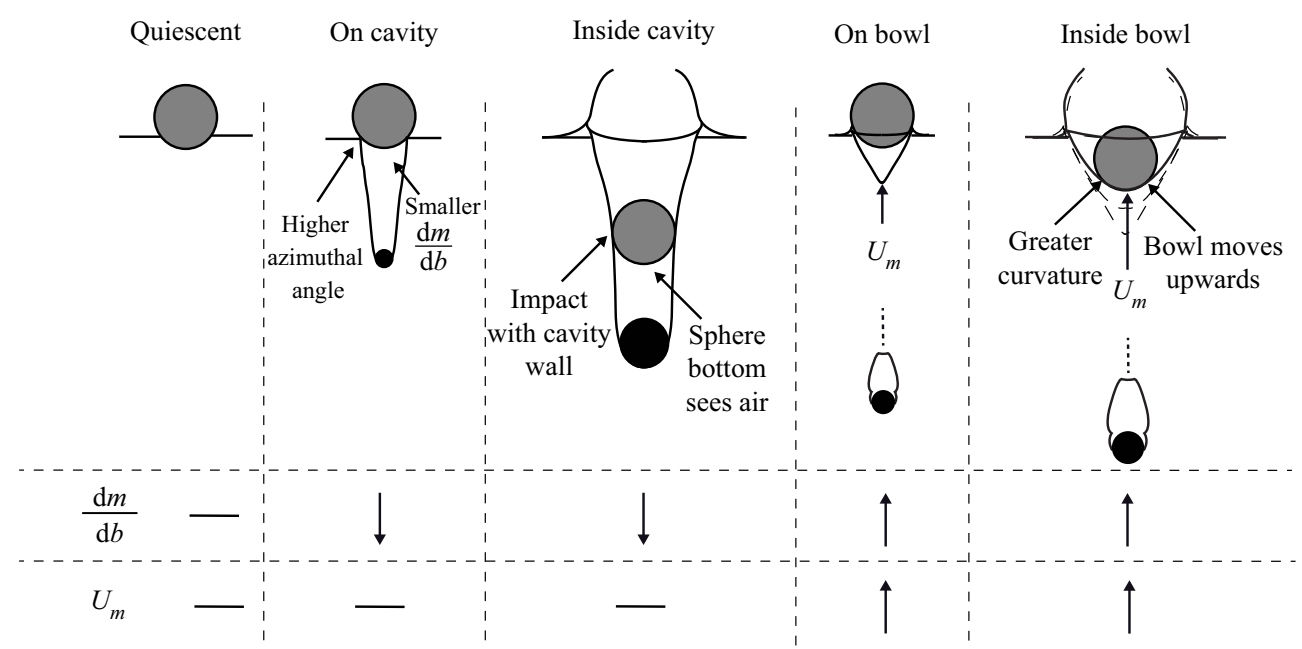

Figure 9. The interaction of the trailing sphere with different stages of the cavity in front leads to varying impact force experienced by the trailing sphere. The impact force depends on the rate of change of added mass $\mathrm{d} m / \mathrm{d} b$, and the upward velocity $U_{m}$ of the added fluid mass. Notional increase/decrease of these values relative to the quiescent case are shown for all but the on jet case (the physics behind which is explained in later discussions).

is larger. It is also possible that the fluid has some upward momentum in the vicinity of the impact location as the cavity evolves toward pinch-off (Truscott et al. 2012; Mansoor et al. 2014), contributing to larger fluid momentum that must be reversed during impact. For the on cavity regime, the cavity diameter at the surface does not vary much in time. Therefore, it seems unlikely that a significant difference in $\mathrm{d} m / \mathrm{d} b$ exists between the on cavity cases. A possible source of the increase in acceleration is the fluid momentum in the vicinity of the cavity opening. For small $M t$, the cavity is expanding and the added fluid momentum is outward. With increasing $M t$, the added fluid momentum becomes neutral as the cavity stops expanding, and thus the second sphere needs to provide a larger change of momentum for the added fluid mass. In general, as $M t$ increases and the two-sphere impacts transition between the regimes depicted in figure 9, the relative contributions from $\mathrm{d} m / \mathrm{d} b$ and $U_{m}$ change. Detailed modelling of the sphere impact with the various cavity states is beyond the scope of this work, but the framework presented here lends insight into how the peak force changes with $M t$ for the observed two-sphere regimes.

At first glance, we would expect both on cavity and inside cavity cases to transition to on bowl and inside bowl cases respectively when $M t \sim 1$. This is true for the on-cavity-to-on-bowl transition. Yet, when $d_{c}>d_{2}$ and $M t<1$, pinch-off can occur while the upper sphere falls through the upper portion of the cavity leading to an in bowl impact. This reduces the $M t$ value for the in-cavity-to-in-bowl transition, which we can predict by taking into account the time $t_{H P}$ for the upper sphere to fall from the original free-surface level to the pinch-off point. We find that the limiting pinch-off time for this to occur is,

$$
t_{p}=\left(t_{2}-t_{1}\right)+t_{H P}
$$

Dividing both sides of (3.6) by $t_{p}$ and rearranging we get,

$$
M t_{c}=1-\frac{t_{H P}}{t_{p}} .
$$




\section{Impact force reduction}

From (3.7) we see that $M t_{c}$ is the critical value where in-cavity-to-in bowl transition would occur, and $M t_{c}$ is smaller than 1 . The time $t_{H P}$ can be estimated using the relation $t_{H P}=$ $\left(u_{2 p}-u_{2}\right) / g$, where $u_{2 p}$ is the velocity with which the upper sphere impacts the pinch-off point and $u_{2}$ is the initial velocity with which the upper sphere passes the free surface $\left(u_{2}=\right.$ $\left.\sqrt{2 g h_{2}}\right)$. The value $u_{2 p}$ can be calculated from the relation $u_{2 p}=\sqrt{u_{2}^{2}+2 g H_{p}}$, where $H_{p}$ is the pinch-off depth; $H_{p}$ can be estimated using the empirical relation $H_{p}=0.57 d_{1} \sqrt{F r}$ (Duclaux et al. 2007), where $d_{1}$ is the lower sphere diameter. Then, (3.7) can be rewritten as,

$$
M t_{c}=1-\frac{\sqrt{u_{2}^{2}+2 g H_{p}}-u_{2}}{g t_{p}}=1-\frac{2\left(\sqrt{h_{2}+0.57 d_{1} F r^{1 / 2}}-\sqrt{h_{2}}\right)}{\beta \sqrt{d_{1}}}
$$

where $t_{p}$ is written as $t_{p}=\beta \sqrt{d_{1} /(2 g)}$. Equation (3.8) reveals the transition $M t_{c}$ at which the upper sphere falls onto the pinch-off singularity from independent parameters $h_{1}, d_{1}$ and $h_{2}$. Using these parameters from 33 different two-sphere cases, this $M t_{c}$ is determined to be $0.6539 \pm 0.022$ (95\% confidence). Plotting this line as the separation criterion for inside cavity and bowl cases in figure 8 illustrates that the experimental data agree with this $M t_{c}=0.65$ separation line quite well.

Depending on the cavity opening diameter $d_{c}$, two different on bowl modes are possible: on cavity bowl $\left(d_{c}<d_{2}\right)$ and inside cavity bowl $\left(d_{c}>d_{2}\right)$. They transition from on cavity and inside cavity modes at $M t \sim 1$ and $\sim 0.65$ respectively as discussed earlier. In both, as discussed with the help of (3.5), the coupled effects of upward added mass velocity $U_{m}$ and the contoured curvature of the bowl surface increasing the $\mathrm{d} m / \mathrm{d} b$ value leads to dramatically higher peak impact accelerations than a normal quiescent case (figure $7(a)$, 0-9 ms, 7(b), 10-20 ms). Figure 8 shows that the bowl cases almost always have higher impact acceleration; in some instances impact forces almost quadruple that of the quiescent case value $(\approx 427 \%$ at $M t \sim 0.96)$. Thus, this $0.65<M t<1.43$ range of Matryoshka values $(M t \sim 1.43$ being the transition from bowl to jet mode, discussed in the next paragraph) is a range to avoid if one wishes to achieve any sort of reduction in impact force. The inside cavity bowl cases show much higher accelerations than their on cavity bowl counterparts, which can be attributed to the upward region of the bowl being much larger for inside cavity bowl cases and hence the average $U_{m}$ and $\mathrm{d} m / \mathrm{d} b$ are greater over the frontal area of the sphere.

The bowl eventually forms an upward moving axial jet called a Worthington jet (Worthington \& Cole 1897; Worthington 1908; Gekle \& Gordillo 2010) coming out of the base of the distorted bowl-shaped free surface following the collapse of the cavity after pinch-off $(M t \geq 1.43)$. Here, the on jet cases are characterized by the upper sphere dramatically passing through the Worthington jet into the water pool (figure 7c). Finding the $M t$ at which the on bowl cases would transition to on jet cases is a complicated task, since theoretical consideration of the bowl shaped free-surface distortion that results in the Worthington jet is scarce in the literature. To estimate the $M t$ where we could expect the transition from bowl to jet mode to happen, we experimentally observed 20 different quiescent drops that result in Worthington jets and calculated the time $t_{j}$ after pinch-off $\left(t_{p}\right)$ for the underwater bowl to transform into a fully formed jet above the free surface, assuming the jet to be fully formed when the upwards moving sub-surface bowl has fully vanished. If the upper sphere passes the free-surface line at the exact moment when the bowl vanishes, we can consider the upper sphere free-surface passing time $t_{2}$ as the onset 


\section{R. Rabbi and others}
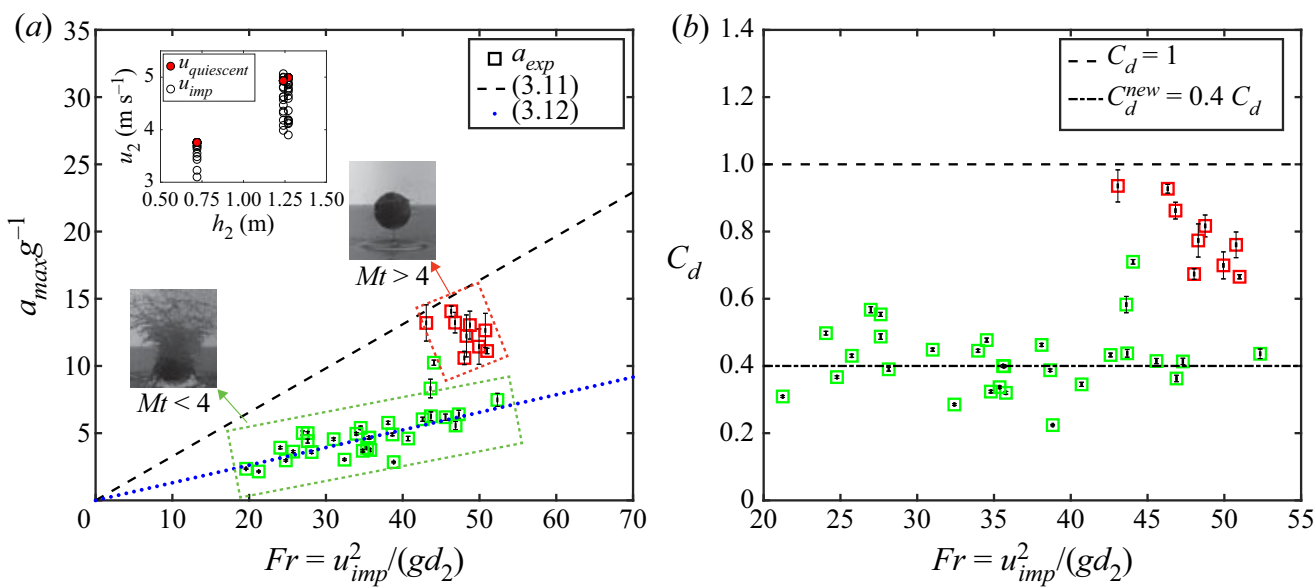

Figure 10. The maximum impact accelerations $(a)$ and the associated impact drag coefficients $C_{d}(b)$ in on jet cases. Prolonged collision with the upward moving jet results in a reduced trailing sphere impact velocity $u_{i m p}$, shown for several cases in the inset in $(a)$. The dashed line in $(a)$ shows the theoretical impact acceleration using this reduced $u_{\text {imp }}$ in (3.11), and the dotted line represents (3.12) with $\alpha=0.4$. The squares plot actual measured impact accelerations in different on jet cases, the green squares are for $M t<4$ cases, and the red squares are for $M t>4$. In $(b)$, the impact drag coefficients are plotted for both $M t<4$ and $M t>4$, with the dashed line showing quiescent impact drag coefficient of 1 , and the dash-dotted line representing $C_{d}^{\text {new }} \sim$ $0.4 C_{d}$. Uncertainty bands are marked with $95 \%$ confidence interval.

of on jet cases. Thus, we can write

$$
\left(t_{2}-t_{1}\right)=t_{p}+t_{j}
$$

to define the transition from bowl to on jet ( $t_{1}$ is the time of the lower sphere impacting the free surface). Now dividing both sides of (3.9) by $t_{p}$, we end up with,

$$
M t=1+\frac{t_{j}}{t_{p}} .
$$

Thus (3.10) finds the $M t$ value of the onset of the on jet mode. From the 20 observations, the fractional term $t_{j} / t_{p}$ is found to be a constant value of $0.43 \pm 0.06$ for varied $F r$ impacts, resulting in the $M t$ value of $1.4345 \pm 0.06$ (standard uncertainty, $95 \%$ confidence, see appendix A.3, figure 17). The value of $M t \sim 1.43$ can be used as the separation criterion between on bowl and on jet modes, and the experimental data presented in figure 8 follow this separation reasonably well.

The acceleration reduction for these $M t \geq 1.43$ cases are also dramatic, with the highest reduction of up to $\sim 78 \%$ observed experimentally $(M t=2.67$, figure 8$)$. The time steps and the acceleration plot presented in figure $7(c)$ provide an explanation of how the reduction in impact acceleration manifests. The jet starts wetting the trailing upper sphere long before it has reached the free surface $(80 \mathrm{~ms}$ before free-surface impact, not shown in figure $7(d)$ on jet case, see figure 18). This drawn out collision with the narrow axial jet results in a reduction in momentum of the upper sphere over a longer period of time, and also results in partial wetting of the upper sphere by the jet. At the time of impact, the water-enveloped upper sphere does not abruptly go through an air-water interface like the quiescent case does. Instead, it enters the water pool through a thick jet base and is enveloped by the jet to such extent that it resembles a sphere inside a thick jet entering the pool. Speirs et al. (2019a) showed that, for a similar kind of water entry of a sphere 


\section{Impact force reduction}

(a)

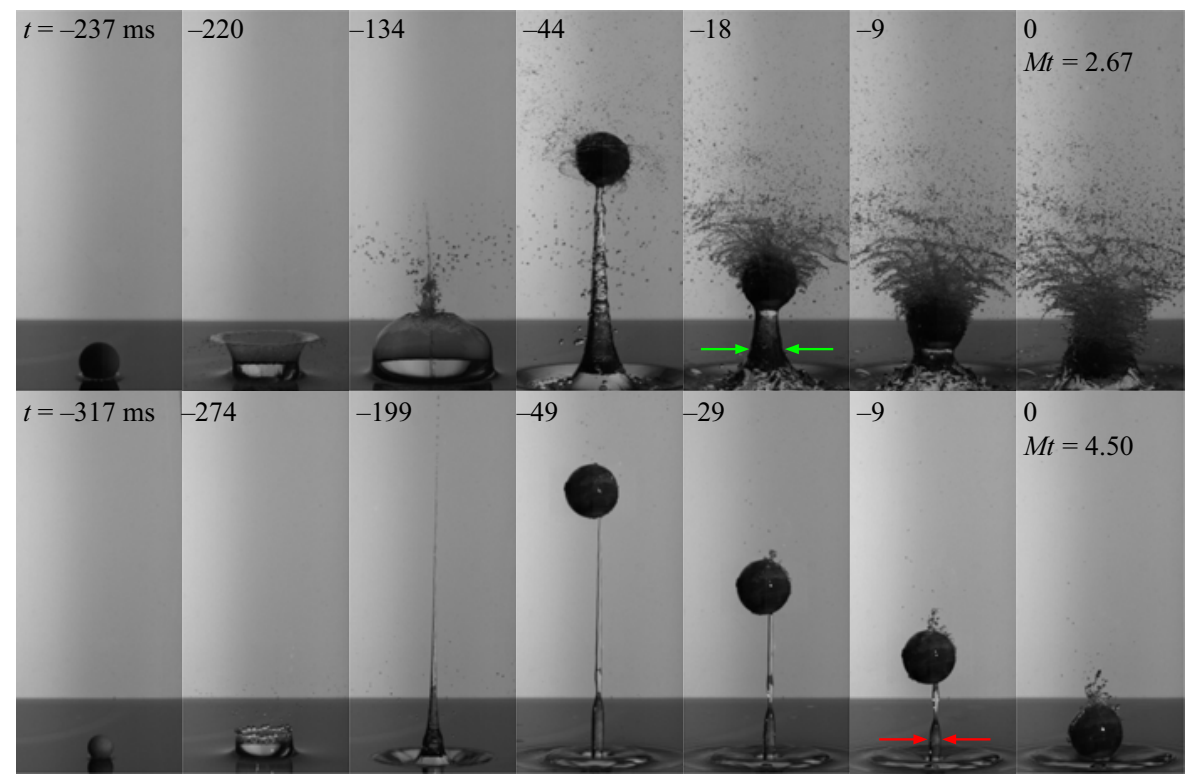

Figure 11. Two different on jet cases happening at either side of $M t \sim 4$ resulting in contrasting impact accelerations. In $(a), M t=2.67$, and the upper sphere falls through a fully formed Worthington jet. The lower sphere diameter is $38 \mathrm{~mm}$. The crash through the jet is violent, the sphere gets entirely enveloped by the jet and falls through a thick jet base with a reduced impact velocity of $4.36 \mathrm{~m} \mathrm{~s}^{-1}$. The acceleration reduction here is prominent, $\sim 78 \%$ for this particular case. (b) An on jet case of $M t=4.50$. The lower sphere $\left(d_{1} \sim 23.75 \mathrm{~mm}\right)$ creates a cavity that evolves into the Worthington jet. Larger $M t$ results in a thinner jet and a significantly calmer impact event when the trailing sphere passes the free surface with a reduced impact velocity $4.89 \mathrm{~m} \mathrm{~s}^{-1}$. The jet base is non-existent, the sphere falls through a retracting water column. The reduction in impact acceleration here is a mere $\sim 7 \%$. There is stark difference in jet diameter $d_{j}$ at the jet base also; $d_{j}$ is much bigger in (a) than in (b).

inside a free-falling jet, the maximum impact acceleration is a function of $F r$, and can be modelled using a modification to the force balance equation,

$$
\frac{a_{q}}{g}=\frac{3}{4} \frac{\rho}{\rho_{s}} C_{d_{\max }} F r,
$$

where $\rho / \rho_{s}$ is the density ratio of water and the sphere $\left(\sim 2.29\right.$ in our study), and $C_{d_{\max }}$ is the impact drag coefficient. Shiffman \& Spencer (1945) showed that, for large density ratios, the value of $C_{d_{\max }}$ can be taken as $\sim 1$ during the initial moment of entry (when $b \sim 0.1-0.2$ per discussion of (3.3)) of a single sphere into a quiescent pool. Speirs et al. (2019a) argued that when a sphere enters the pool travelling within a jet, this $C_{d_{\max }} \sim 1$ value decreases, and (3.11) becomes,

$$
\frac{a}{g}=\frac{3}{4} \frac{\rho}{\rho_{s}}\left(\alpha C_{d_{\max }}\right) F r
$$

where they reported $\alpha=0.25$, reducing the impact drag coefficient in sphere in a jet cases to $C_{d}^{\text {new }}=0.25 C_{d_{\max }}$. Despite the opposing jet direction between Speirs et al. (2019a) and the Worthington jets in this study, plotting the measured impact acceleration values as a function of $\mathrm{Fr}$ in figure 10 reveals a similar reduction in impact drag coefficient for the on jet cases, albeit only in the $1.43 \leq M t<4$ range. We experimentally observe $\alpha \sim$ 


\section{R. Rabbi and others}

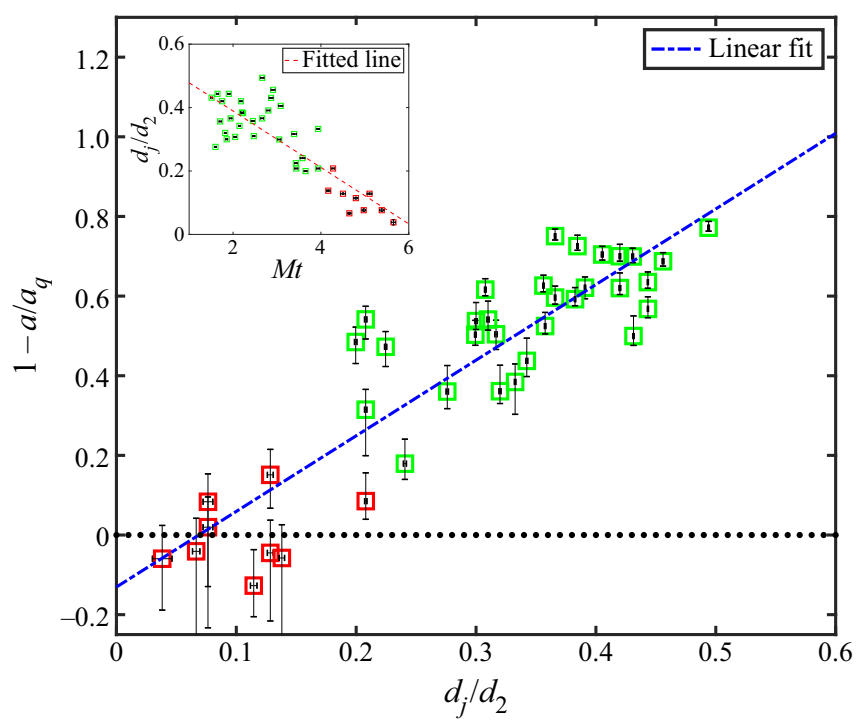

Figure 12. The reduction in impact acceleration in on jet cases are linearly dependent on the jet base diameter prior to the upper sphere passing the free-surface line. Increasing jet diameter results in higher reduction, with the jet diameter monotonically decreasing over increasing $M t$, as shown in the inset. The different coloured markers follow the same convention presented in figure 10. The blue dashed line is fitted through least-square regression giving the equation: $1-a / a_{q}=1.89\left(d_{j} / d_{2}\right)-0.1306$.

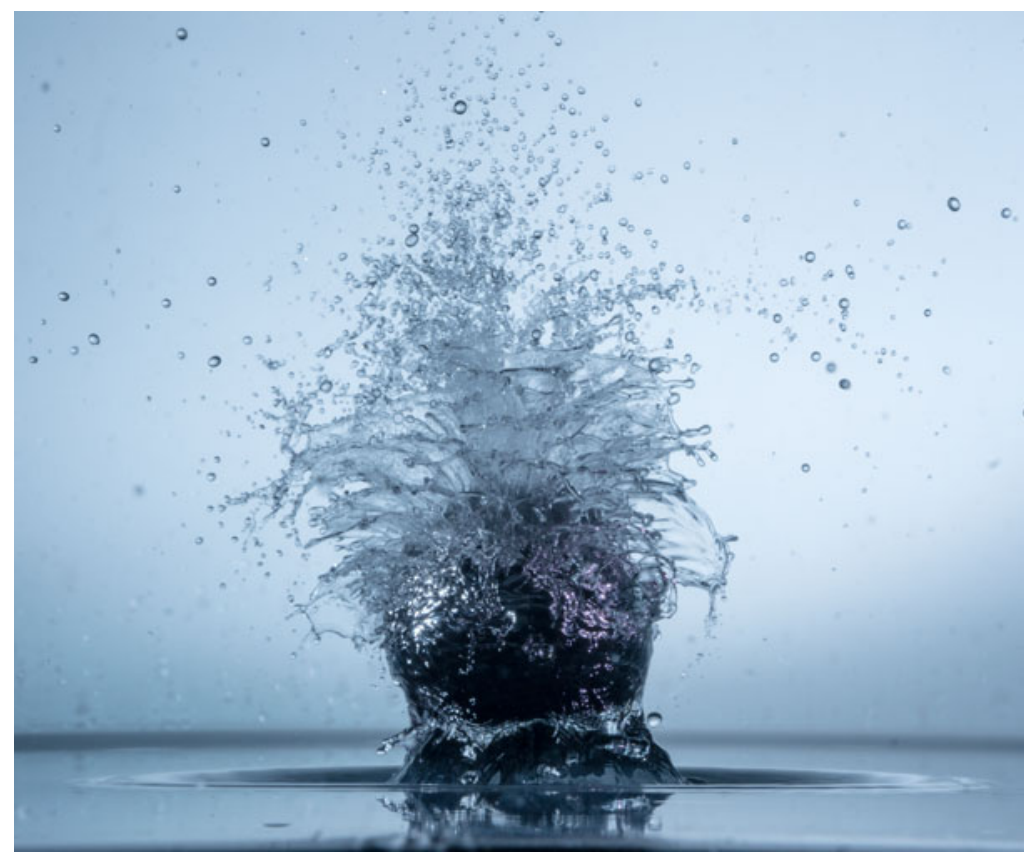

Figure 13. A $50 \mathrm{~mm}$ steel sphere falling through the Worthington jet originating from the collapse of a cavity created by a $38 \mathrm{~mm}$ hydrophobic steel sphere impacting a quiescent water pool at $2.09 \mathrm{~m} \mathrm{~s}^{-1}$. The beautiful fluid patterns observed at the surface of the sphere originate from the jet water films following the sphere curvature and crashing together at the top of the sphere. 


\section{Impact force reduction}

0.4 , yielding $C_{d}^{\text {new }}=0.4 C_{d_{\max }}$ (figure $10 a, b$ ) for $M t<4$ on jet cases, with the Froude of impact calculated from $\mathrm{Fr}=u_{\text {imp }}^{2} /\left(g d_{2}\right)$, where $u_{i m p}$ is the reduced impact velocity resulting from the sustained collision of the upper sphere with the Worthington jet (inset, figure 10a). At $M t>4$, the on jet $C_{d}$ values are closer to one (figure $10 b$ ), which can be attributed to the retreating nature of the Worthington jet as $M t$ increases. A greater $M t$ indicates a larger time difference between the jet creation and the upper sphere passing the free surface. Whereas thick jet diameters and prominent jet bases are typical of $M t<4$ cases (figure 11a), as the time between the two-sphere free-surface impact is increased, the Worthington jet changes from an upward rising jet to a descending jet, with the jet peak starting to thin, and the jet base eventually vanishing beyond $M t \sim 4$ (figure $11 b$ ).

We can understand how the jet base diameter affects impact acceleration by again considering the rate of change of added mass $d m / d b$. As the sphere moves through the jet, some amount of the jet fluid passes around the sphere, but presumably some amount is also accelerated downward by the sphere. As the sphere descends, the jet diameter increases smoothly to the value $d_{j}$ (measured at the top of the jet base when the sphere is one diameter above the base as shown in figure 11). One might expect the fluid below the flat water surface to be accelerated by the downward moving jet fluid prior to the sphere entering the water pool. This effect would reduce the value of $\mathrm{d} m / \mathrm{d} b$ during water entry of the sphere, thus reducing the impact force and resultant acceleration. As $d_{j} \longrightarrow d_{2}$ and a larger amount of fluid mass exists in the jet base, $\mathrm{d} m / \mathrm{d} b$ would be smaller at the moment of water entry, and one would expect the impact acceleration reduction to be greater. This notion is supported by the data in figure 12 , which show acceleration reduction increasing with increasing $d_{j} / d_{2}$.

When the second sphere falls through a thick Worthington jet a chaotic but pretty watery mess can occur, as shown in figure 13. As the second sphere falls through the jet, it forces it into a thin film, prompting intriguing fingering patterns, buckling and droplets at the top of the sphere as the thin film envelops the sphere over the apex. A time series showing this violent yet beautiful splash phenomenon can also be seen in figure 11(a).

In some of the on jet cases, the Worthington jet created from pinch-off is suppressed by the splash crown domeover at the free surface. This results in a disturbed, jet-like water column at the free surface, through which the upper sphere falls. Here, we consider these cases as 'on surface jet' cases, since their formation mechanism is different from that of the on jet cases (see appendix A.4, figure 19). These cases show reduction in acceleration similar to the on jet cases as illustrated in figure 8 .

\section{Conclusion}

The impulse force felt by any object at the initial moment of water impact can be very high (figure 1c), and may prove to be catastrophic for water landing craft or missiles, and fatal even for thrill seeking bungee jumpers (Von Karman 1929; McGehee et al. 1959; Kornhauser 1964; Thompson 1965). Herein, we have shown through a canonical sphere impact study that the initial impact force can be greatly reduced by first launching another object in front of the body of interest. The force of impact is reduced by the cavity of the first object providing less initial water impact area, lower relative velocities or upward jets that wet and decelerate the trailing body. However, if the object encounters the collapsing upward cavity, the upper sphere may experience a larger force of impact than if the leading object were not present at all. A non-dimensional number called the Matryoshka number $M t$ is defined to classify two-sphere consecutive water entry behaviour based on the object size and the cavity pinch-off time. Experimental results show that for $0.2<M t<0.65$ and 


\section{R. Rabbi and others}

$1.43<M t<4$, significant reduction of the impact acceleration of the trailing sphere is achieved. In the interim range of $0.65<M t<1.43$, a sudden rise in impact acceleration is observed, and must be avoided if trying to avoid catastrophic failures. This $M t$ formulation can potentially be used to predict interactions in any multi-object water entry system, and the regime diagram proposed in figure 8 does well to aid in making predictions for size differences and timing.

Supplementary movies. Supplementary movies are available at https://doi.org/10.1017/jfm.2020.1165.

Funding. R.R., N.S., J.B. and T.T.T. acknowledge funding from the Office of Naval Research, Navy Undersea Research Program (Grant no. N000141812334), monitored by Ms M. Medeiros. A.K. is a JSPS Overseas Research Fellow.

Declaration of interests. The authors report no conflict of interest.

\section{Author ORCIDs.}

(D) Rafsan Rabbi https://orcid.org/0000-0002-4799-1855;

D Akihito Kiyama https://orcid.org/0000-0001-6205-044X;

D Tadd T. Truscott https://orcid.org/0000-0003-1613-6052.

\section{Appendix A}

\section{A.1. Uncertainty analysis}

To calculate the uncertainty relating to the variables measured for our two-sphere study we first look at our reported results. From figures $6(c), 7(d)$ and 8 in the main text it is evident that we need to consider the uncertainty relating to measured acceleration peaks and the reported $M t$ numbers by propagating uncertainty through the acceleration reduction equation $\left(1-a / a_{q}\right)$ and the $M t$ number formulation (3.1).

First, we propagate the uncertainty through $M t$ formulation,

$$
\begin{aligned}
M t & =\frac{t_{2}-t_{1}}{t_{p}} \\
& =\frac{t_{2}-t_{1}}{\beta \sqrt{\frac{d_{1}}{2 g}}},
\end{aligned}
$$

where $d_{1}$ is the sphere diameter and $g$ is the gravitational constant. Applying the Taylor series method (TSM) approach to propagate uncertainty,

$$
u_{M t}^{2}=\left(\frac{\partial M t}{\partial\left(t_{2}-t_{1}\right)}\right)^{2} u_{t_{2}-t_{1}}^{2}+\left(\frac{\partial M t}{\partial \beta}\right)^{2} u_{\beta}^{2}+\left(\frac{\partial M t}{\partial d_{1}}\right)^{2} u_{d_{1}}^{2} .
$$

Now, the partial derivatives of $M t$ with respect to the variables are,

$$
\frac{\partial M t}{\partial\left(t_{2}-t_{1}\right)}=\frac{1}{\beta \sqrt{\frac{d_{1}}{2 g}}}, \quad \frac{\partial M t}{\partial \beta}=-\frac{\left(t_{2}-t_{1}\right)}{\beta^{2} \sqrt{\frac{d_{1}}{2 g}}}, \quad \frac{\partial M t}{\partial R}=-\left(\frac{2}{d}\right)^{1.5} \frac{\left(t_{2}-t_{1}\right) \sqrt{g}}{\beta} .
$$

$(\mathrm{A} 3 a-c)$

The values $\left(t_{2}-t_{1}\right)$, sphere diameter $d_{1}$ and $\beta$ are experimentally measured values all of which has uncertainty associated with them. These uncertainties can be reported as,

$$
u_{\left(t_{2}-t_{1}\right)}=0.05 \times 10^{-3}, \quad u_{\beta}=0.0497, \quad u_{d_{1}}=0.02 \times 10^{-3} .
$$

$(\mathrm{A} 4 a-c)$ 


\section{Impact force reduction}

Using these and substituting the partial derivatives of (A2) by (A3) we get,

$$
\begin{aligned}
u_{M t}^{2}= & \left(\frac{\sqrt{2 g}}{2.03 \sqrt{d_{1}}} 0.5 \times 10^{-3}\right)^{2}+\left(\frac{\left(t_{2}-t_{1}\right)}{2.03^{2} \sqrt{\frac{d_{1}}{2 g}}} 0.0497\right)^{2} \\
& +\left(\frac{\left(t_{2}-t_{1}\right) \sqrt{g}}{2.03\left(\frac{d_{1}}{2}\right)^{3 / 2}} 0.02 \times 10^{-3}\right)^{2} .
\end{aligned}
$$

Thus (A5) gives the uncertainty for individual $M t$ numbers explored in the study.

We can propagate uncertainty in the same manner for the acceleration reduction equation,

$$
R=1-\frac{a}{a_{q}},
$$

where $R$ denotes the amount of reduction experienced for a two-sphere case, $a_{q}$ is the quiescent acceleration and $a$ is the two-sphere acceleration for any particular case. Propagating uncertainty through TSM,

$$
u_{R}^{2}=\left(\frac{\partial R}{\partial a}\right)^{2} u_{a}^{2}+\left(\frac{\partial R}{\partial a_{q}}\right)^{2} u_{a_{q}}^{2} .
$$

Now, the individual uncertainties associated with the acceleration measurement come from the device uncertainty and the low sampling rate of the accelerometer $(1000 \mathrm{~Hz})$. The uncertainties relating to measured accelerations $\left(u_{a_{q}}\right.$ and $\left.u_{a}\right)$ are one sided, assuming the low sampling rate of the accelerometer fails to capture the exact highest peak of any acceleration event. Applying these uncertainties coupled with device uncertainty itself, we see that we would have two-sided uncertainty, where $u_{a_{q}}$ will drive the upper uncertainty for any data point since a bigger $a_{q}$ will increase the reduction value in (A6), and $u_{a}$ drives the lower uncertainty since a greater impact acceleration $a$ will reduce the amount of acceleration reduction. Taking these into account, we can rewrite (A7) as a two-sided uncertainty where,

$$
\begin{gathered}
u_{\text {up }}^{2}=\left(\frac{\partial R}{\partial a}\right)^{2} \times\left(u_{\text {device }}\right)^{2}+\left(\frac{\partial R}{\partial a_{q}}\right)^{2} \times\left(\sqrt{u_{\text {device }}^{2}+u_{a_{q}}^{2}}\right)^{2} \\
u_{\text {down }}^{2}=\left(\frac{\partial R}{\partial a}\right)^{2} \times\left(\sqrt{u_{\text {device }}^{2}+u_{a}^{2}}\right)^{2}+\left(\frac{\partial R}{\partial a_{q}}\right)^{2} \times\left(u_{\text {device }}\right)^{2} .
\end{gathered}
$$

The partial derivative terms from (A8) and (A9) can be written as,

$$
\frac{\partial R}{\partial a}=-\frac{1}{a_{q}}, \quad \frac{\partial R}{\partial a_{q}}=\frac{a}{a_{q}^{2}} .
$$

Device uncertainty $u_{\text {device }}$ can be calculated from the datasheets of the accelerometers used (MPU 9250A manufactured by Invensen Inc. and H3LIS331DL produced by STMicroelectronics). For calculating $u_{a}$ and $u_{a_{q}}$, we employed a different method. 
(a)

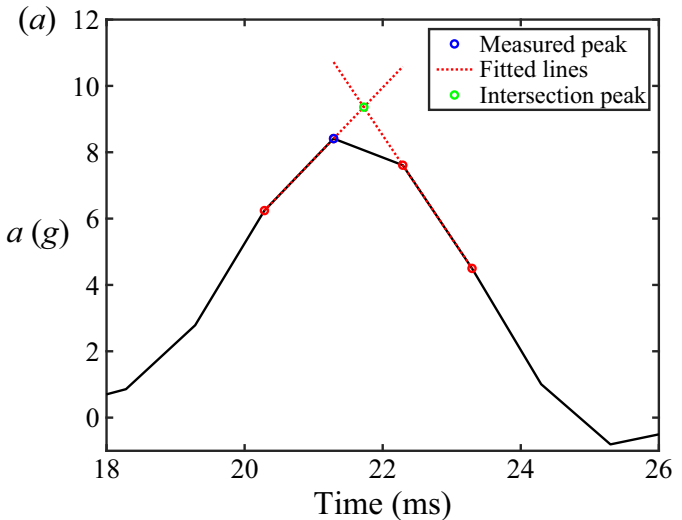

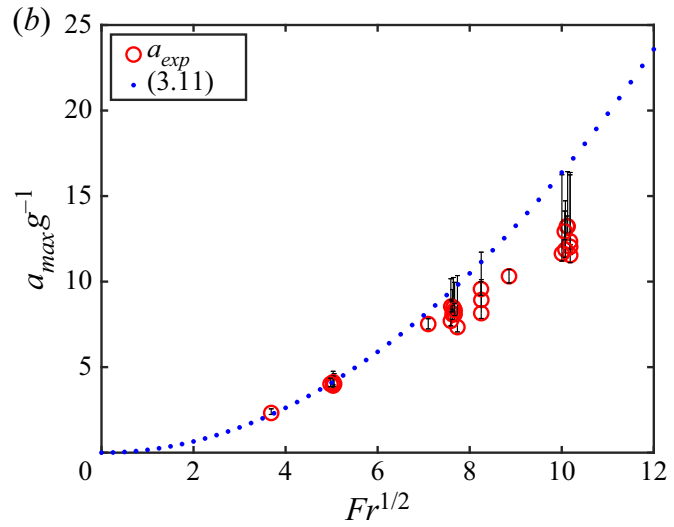

Figure 14. (a) Peak estimation using the line intersection method. The true peak should lie between the two highest measured peak values. Fitting two straight lines to these two data points results in an intersection point (denoted by the green circle) higher in magnitude than both the highest points and is in between the points also. The difference between the measured peak (blue circle in the plot) and the estimated peak (green circle) can be taken as the uncertainty value for this two-sphere impact event. (b) Measured quiescent acceleration data compared with theoretical estimations calculated using (3.11). At lower $F r$, the measured acceleration follows (3.11). With increasing $F r$, underestimation of peak acceleration may happen, but it happens mostly within the uncertainty bounds.

Based on the assumption that the low sampling rate of $1000 \mathrm{~Hz}$ is not enough to capture the highest point of impact acceleration, we assumed that the highest acceleration point for any two-sphere impact event would reside between the two highest points physically measured by the accelerometer. Then we fit two straight lines comprising of the two maximum points and a few other points on both sides of the true peak acceleration point. The lines would intersect in between the two measured highest acceleration point, and the difference between this intersection peak and measured peak will be our acceleration uncertainty $u_{a_{q}}$ and $u_{a}$. Figure 14(a) illustrates this idea of estimating the uncertainty relating to the true peak acceleration. Figure 14(b) shows the application of the uncertainty bands calculated this way to experimentally measured quiescent peak acceleration data. The measured value underestimates the peak values when compared to theoretical peak values (calculated using (3.11) with $C_{d} \sim 1$ ) with increasing $F r$, but falls within the uncertainty bounds.

Using this approach, we estimated the peak acceleration values for each of the data points presented in figure 8 . This also gives us an idea of how much uncertainty we are dealing with for the individual acceleration curves reported in figures $6(c)$ and $7(d)$. Applying the calculated uncertainty values for $u_{a_{q}}$ and $u_{a}$ and using (A8) and A9 we measured two-sided uncertainty for all the reported cases from figure 8 . Figure 8 in the main text plots all the data points with their uncertainty bands presented.

\section{A.2. Impact force dependence on added mass}

The analysis here follows from Shiffman \& Spencer (1945) and May (1975), who essentially report the same analysis. For impact of a sphere on a quiescent free surface, their analysis starts with the notion that momentum is conserved during a water impact such that

$$
M U_{o}=(M+m) U,
$$

where $M$ is the sphere mass, $m$ is the added mass of the fluid, $U_{o}$ the impact velocity and $U$ the instantaneous velocity of the sphere and added fluid mass after impact. As the force 


\section{Impact force reduction}

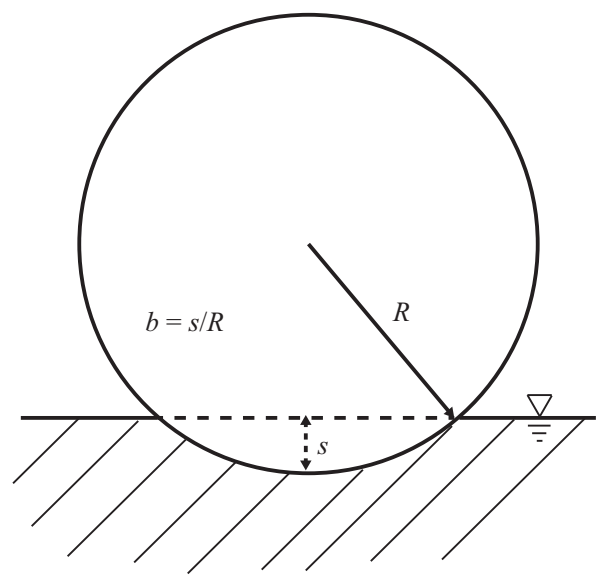

Figure 15. Schematic diagram of a sphere penetrating the free surface.
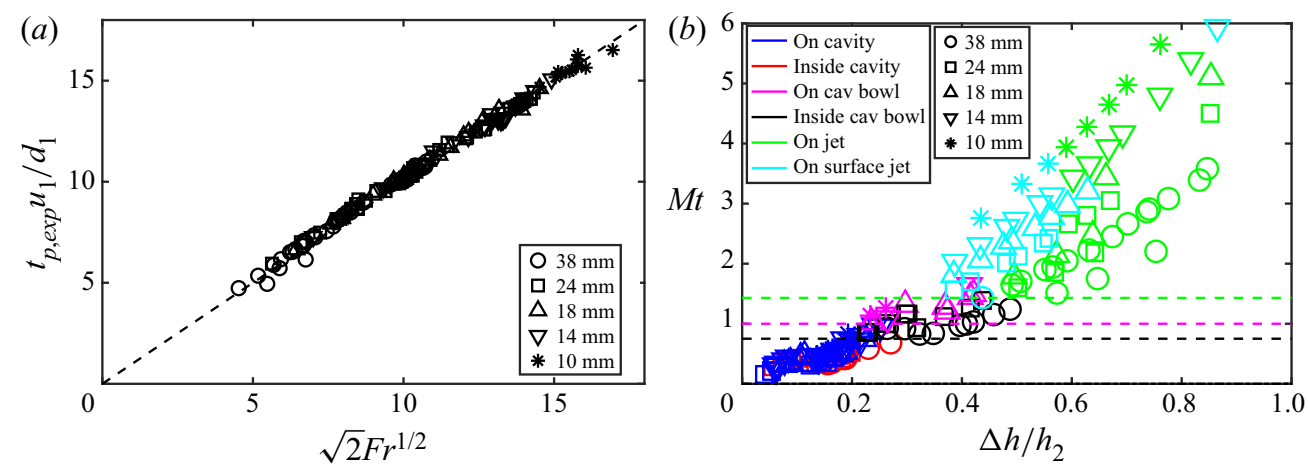

Figure 16. (a) The non-dimensional pinch-off time $t_{p} u_{1} / d_{1}$ can be scaled with $\sqrt{2} F r^{1 / 2}$, which agrees with previous such reports in the literature (Aristoff et al. 2010). (b) The relation between $M t$ and non-dimensional spacing between the spheres show that, for the same spacing between different diameter spheres, $M t$ will be different, leading to different reduction of impact forces. The on surface jets are cases which can be treated as extension to on jet cases.

due to added mass dominates forces in the early stages of impact for the regime of our study, this force is given by

$$
F_{z}=-M \frac{\mathrm{d} U}{\mathrm{~d} t} .
$$

Solving (A11) for $U$ and taking the derivative with respect to time gives

$$
\frac{\mathrm{d} U}{\mathrm{~d} t}=-U_{o} \frac{M}{(m+M)^{2}} \frac{\mathrm{d} m}{\mathrm{~d} t} .
$$

The time derivative can be converted to a submergence-dependent derivative by first defining $b=s / R$, where $R$ is the sphere radius and $s$ the submergence below the initial free-surface plane (figure 15). Derivatives are related by

$$
\frac{\mathrm{d}()}{\mathrm{d} t}=\frac{\mathrm{d}()}{\mathrm{d} b} \frac{\mathrm{d} b}{\mathrm{~d} s} \frac{\mathrm{d} s}{\mathrm{~d} t}=\frac{U}{R} \frac{\mathrm{d}()}{\mathrm{d} b} .
$$




\section{R. Rabbi and others}
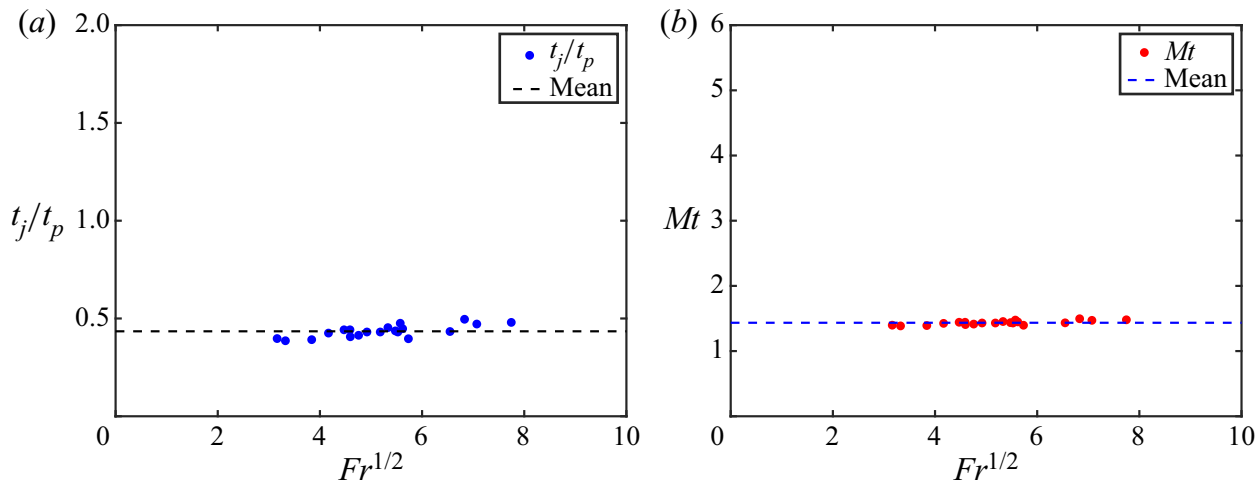

Figure 17. The time the upwards moving bowl takes to evolve into a fully fledged Worthington jet from the moment of pinch-off is invariant with the impact parameters $(a)$, resulting in $M t=1.43$ as the criterion for transition from bowl modes to on jet modes $(b)$.

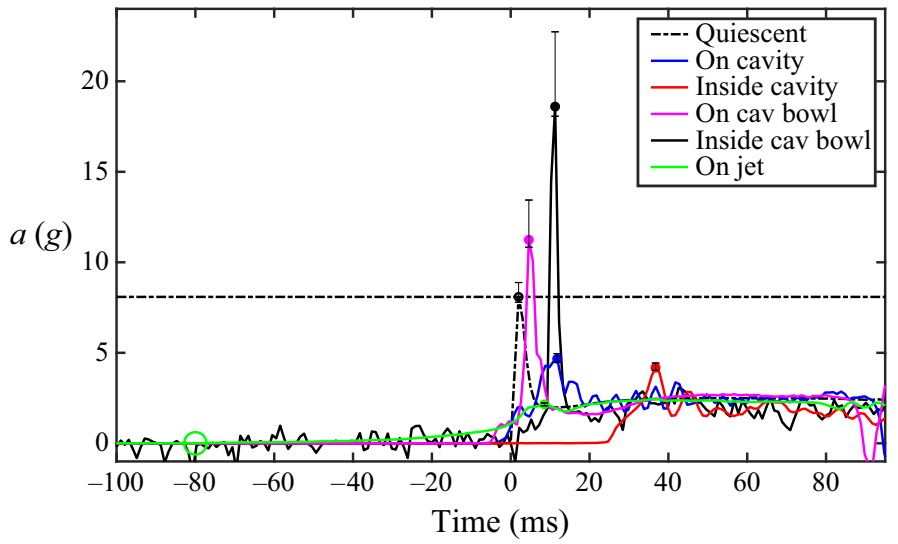

Figure 18. Acceleration plot for different two-sphere water entry cases. This plot shows the acceleration felt by the free-falling sphere, going back $100 \mathrm{~ms}$ before free-surface impact. In the on jet case shown by the green line, the sphere meets the upward moving Worthington jet significantly ahead in time, $\approx 80 \mathrm{~ms}$ before impact in the case presented here, the green circle in the plot denotes the approximate time when the sphere meets the jet.

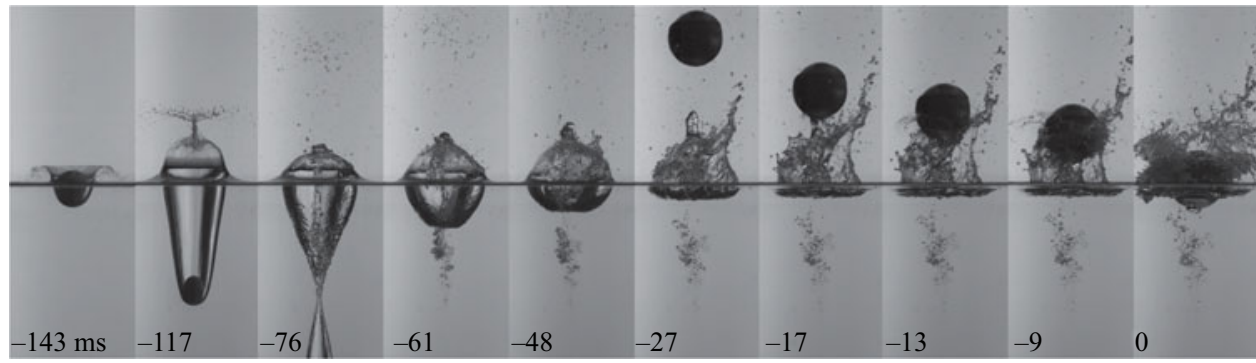

Figure 19. A consecutive two-sphere impact event with $M t=1.99$. A $23.75 \mathrm{~mm}$ sphere impacts the free surface at a velocity of $\sim 3.6 \mathrm{~m} \mathrm{~s}^{-1}$ creating a cavity. The cavity domes over at the surface (29 ms) that suppresses the axial jet originating from pinch-off (70-119 ms). This results in a smaller jet at the free surface through which the upper sphere falls (129-146 ms). Similar cases are termed as on surface jet cases. 


\section{Impact force reduction}

Combining (A11)-(A14) and simplifying gives

$$
F_{z}=\frac{U_{o}^{2}}{R} \frac{1}{\left(1+\frac{m}{M}\right)^{3}} \frac{\mathrm{d} m}{\mathrm{~d} b} .
$$

Using the typical definition $F_{z}=\rho \pi R^{2} C_{d} / 2$, where $C_{d}$ is the impact drag coefficient, gives

$$
C_{d}=\frac{2}{\rho \pi R^{3}} \frac{1}{\left(1+\frac{m}{M}\right)^{3}} \frac{\mathrm{d} m}{\mathrm{~d} b},
$$

which Shiffman \& Spencer (1945) report for values of $m / M$. We can see from (A16) that the vertical force varies linearly with the rate of change of added mass with submergence.

For the on bowl and inside bowl cases, the cavity has pinched off and the fluid has significant upward momentum as the bowl retracts. We can rationalize this effect to first order by again considering conservation of momentum before and after impact

$$
M U_{o}-m U_{m}=(M+m) U,
$$

where $U_{m}$ is the average velocity of the added fluid mass just before impact (assumed to be in the direction opposite of $U_{o}$ ). We follow the same derivation for impact on a quiescent free surface as above. Solving (A15) for $U$ and differentiating with respect to time gives

$$
\frac{\mathrm{d} U}{\mathrm{~d} t}=-\left(U_{o}+U_{m}\right) \frac{M}{(m+M)^{2}} \frac{\mathrm{d} m}{\mathrm{~d} t} .
$$

Applying (A14) yields

$$
\frac{\mathrm{d} U}{\mathrm{~d} t}=-\frac{M\left(M U_{o}-m U_{m}\right)}{(m+M)^{3}} \frac{\left(U_{o}+U_{m}\right)}{R} \frac{\mathrm{d} m}{\mathrm{~d} b} .
$$

Finally, inserting (A19) into (A12) and simplifying gives

$$
F_{z}=\frac{\left(U_{o}-\frac{m}{M} U_{m}\right)}{\left(1+\frac{m}{M}\right)^{3}} \frac{\left(U_{o}+U_{m}\right)}{R} \frac{\mathrm{d} m}{\mathrm{~d} b} .
$$

Now the vertical force depends not only on $\mathrm{d} m / \mathrm{d} b$, but also has a first-order dependence on $U_{m}$.

\section{A.3. Transition from on bowl to on jet mode}

Figure $17(a, b)$ shows that the non-dimensional time of the Worthington bowl evolving into a fully fledged Worthington jet does not vary with increasing Fr. Employing the mean $t_{j} / t_{p}$ value in (3.10) gives us the transition $M t \sim 1.43$, which acts as an excellent separation criterion between the bowl mode and the on jet mode (figure 8).

\section{A.4. Surface jet cases and expansion of reduction regime}

For a few of the higher Fr cases, the splash crown domeover at the surface suppresses the Worthington jet. This suppression leads to the creation of a not so high jet-like water column at the free surface. The upper sphere falls through this jet into the water pool. One such example is given in figure 19, where an $M t=1.99$ impact event is presented. 


\section{R. Rabbi and others}

Parameter

1. Lower sphere diameter

2. Upper sphere diameter

3. Drop height (lower sphere)

4. Drop height (upper sphere)

5. Impact velocity (lower sphere)

6. Impact velocity (upper sphere)

Symbol
$d_{1}$
$d_{2}$
$h_{1}$
$h_{2}$
$u_{1}$
$u_{2}$

Value

Unit

$10,14,18,23.75,38$ 50

$0.07-1.24$

$0.72-1.27$

$1.17-4.93$

3.76-4.99 $\mathrm{mm}$

$\mathrm{mm}$

$\mathrm{m}$

$\mathrm{m}$

$\mathrm{m} \mathrm{s}^{-1}$

$\mathrm{m} \mathrm{s}^{-1}$

Table 1. Parameter space for consecutive two-sphere water entry.

\section{REFERENCES}

Aristoff, J.M. \& Bush, J.W.M. 2009 Water entry of small hydrophobic spheres. J. Fluid Mech. 619, 45-34.

Aristoff, J.M., Truscott, T.T., Techet, A.H. \& Bush, J.W.M. 2010 The water entry of decelerating spheres. Phys. fluids 22 (3), 032102.

Bergmann, R., Van Der Meer, D., Gekle, S., Van Der Bos, A. \& Lohse, D. 2009 Controlled impact of a disk on a water surface: cavity dynamics. J. Fluid Mech. 633, 381-409.

Coleman, H.W. \& Steele, W.G. 2018 Experimentation, Validation, and Uncertainty Analysis for Engineers. John Wiley \& Sons.

Duclaux, V., Caillé, F., Duez, C., YBert, C., Bocquet, L. \& Clanet, C. 2007 Dynamics of transient cavities. J. Fluid Mech. 591, 177-19.

Duez, C., Ybert, C., Clanet, C. \& Bocquet, L. 2007 Making a splash with water repellency. Nat. Phys. 3 (3), 180-183.

Elhimer, M., Jacques, N., Alaoui, A.E.M. \& Gabillet, C. 2017 The influence of aeration and compressibility on slamming loads during cone water entry. J. Fluids Struct. 70, 24-46.

GeKLe, S. \& GoRdillo, J.M. 2010 Generation and breakup of worthington jets after cavity collapse. Part 1. Jet formation. J. Fluid Mech. 663, 293-330.

Glasheen, J.W. \& McMahon, T.A. 1996 Vertical water entry of disks at low froude numbers. Phys. Fluids 8 (8), 2078-2083.

GRADY, R.J. 1979 Hydroballistics design handbook. Naval Sea Systems command Hydromechanics Committee, January.

Guillet, T., Mouchet, M., Belayachi, J., Fay, S., Colturi, D., Lundstam, P., Hosoi, P., Clanet, C. \& CoHEN, C. 2020 The hydrodynamics of high diving. In Multidisciplinary Digital Publishing Institute Proceedings, 13th Conf. of the International Sports Engineering Association. vol. 49, p. 73. MDPI.

GüZEL, B. \& KORKMAZ, F.C. 2020 Reducing water entry impact loads on marine structures by surface modification. Brodogradnja 71 (1), 1-18.

Hurd, R., Fanning, T., Pan, Z., Mabey, C., Bodily, K., Hacking, K., Speirs, N. \& Truscott, T. 2015 Matryoshka cavity. Phys. Fluids 27 (9), 091104.

KORNHAUSER, M. 1964 Structural Effects of Impact. Spartan Books.

Korobkin, A.A. \& Pukhnachov, V.V. 1988 Initial stage of water impact. Annu. Rev. Fluid Mech. 20 (1), 159-185.

LI, T. \& SigimuRA, T. 1967 Study of apollo water impact. volume 1-hydrodynamic analysis of apollo water impact final report. Tech. Rep. SID 67-498. North American Aviation Inc.

Mansoor, M.M., Marston, J.O., VAKarelski, I.U. \& Thoroddsen, S.T. 2014 Water entry without surface seal: extended cavity formation. J. Fluid Mech. 743, 295-326.

Marston, J.O., VAKarelski, I.U. \& Thoroddsen, S.T. 2012 Cavity formation by the impact of leidenfrost spheres. J. Fluid Mech. 699, 465-488.

MAY, A. 1970 Review of water-entry theory and data. J. Hydronaut. 4 (4), 140-142.

MAY, A. 1975 Water entry and the cavity-running behavior of missiles. Tech. Rep. Navsea Hydroballistics Advisory Committee Silver Spring Md.

McGehee, J.R., Hathaway, M.E. \& Vaughan, V.L. JR. 1959 Water-landing characteristics of a reentry capsule. Tech. Rep. NASA-MEMO-5-23-59L. NASA Langley Research Center.

Moghisi, M. \& SQuire, P.T. 1981 An experimental investigation of the initial force of impact on a sphere striking a liquid surface. J. Fluid Mech. 108, 133-146. 


\section{Impact force reduction}

QI, D., Feng, J., XU, B., Zhang, J. \& LI, Y. 2016 Investigation of water entry impact forces on airborne-launched AUVs. Engng Appl. Comp. Fluid Mech. 10 (1), 473-484.

Seddon, C.M. \& MoAtamedi, M. 2006 Review of water entry with applications to aerospace structures. Intl J. Impact Engng 32 (7), 1045-1067.

Sharker, S.I., Holekamp, S., Mansoor, M.M., Fish, F.E. \& TruscotT, T.T. 2019 Water entry impact dynamics of diving birds. Bioinspir. Biomim. 14 (5), 056013.

Shiffman, N. \& SpenCER, D.C. 1945 The force of impact on a sphere striking a water surface. Tech. Rep. AMG-NYU-133. Courant Institution of Mathematical Sciences, New York University.

Speirs, N.B., Belden, J., Pan, Z., Holekamp, S., Badlissi, G., Jones, M. \& Truscott, T.T. $2019 a$ The water entry of a sphere in a jet. J. Fluid Mech. 863, 956-968.

Speirs, N.B., Mansoor, M.M., Belden, J. \& TruscotT, T.T. $2019 b$ Water entry of spheres with various contact angles. J. Fluid Mech. 862, R3.

Speirs, N.B., PAn, Z., Belden, J. \& TruscotT, T.T. 2018 The water entry of multi-droplet streams and jets. J. Fluid Mech. 844, 1084-1111.

Thompson, F.L. 1928 Water-pressure distribution on seaplane float. Tech. Rep. 290. National Advisory Committee for Aeronautics.

Thompson, W.C. 1965 Dynamic Model Investigation of the Landing Characteristics of a Manned Spacecraft, vol. 2497. National Aeronautics and Space Administration.

Truscott, T.T., Epps, B.P. \& Belden, J. 2014 Water entry of projectiles. Annu. Rev. Fluid Mech. 46 (1), $355-378$.

TruscotT, T.T., EPPS, B.P. \& TEChET, A.H. 2012 Unsteady forces on spheres during free-surface water entry. J. Fluid Mech. 704, 173-210.

TruscotT, T.T \& TeCheT, A.H. 2009 Water entry of spinning spheres. J. Fluid Mech. 625, 135-165.

Von Karman, T. 1929 The Impact on Seaplane Floats During Landing. National Advisory Committee on Aeronautics.

WAnG, J., FAltinsen, O.M. \& LuGni, C. 2019 Unsteady hydrodynamic forces of solid objects vertically entering the water surface. Phys. Fluids 31 (2), 027101.

Wang, J., Lugni, C. \& FAltinsen, O.M. 2015 Analysis of loads, motions and cavity dynamics during freefall wedges vertically entering the water surface. Appl. Ocean Res. 51, 38-53.

Watanabe, S. 1933 Resistance of impact on water surface, part v-sphere. Sci. P. Inst. Phys. Chem. Res. 23 (484), 202-209.

Worthington, A.M. 1908 A Study of Splashes. Longmans Green \& Co. (see also 1963 reprint, Macmillan).

Worthington, A.M. \& Cole, R.S. 1897 Impact with a liquid surface, studied by the aid of instantaneous photography. Phil. Trans. R. Soc. Lond. A 189, 137-148.

Yang, G., Vaikuntanathan, V., Terzis, A., Cheng, X., Weigand, B. \& Helmig, R. 2019 Impact of a linear array of hydrophilic and superhydrophobic spheres on a deep water pool. Colloid. Inter. 3 (1), 29.

YUN, H., LYU, X. \& WEI, Z. 2020 Experimental study on oblique water entry of two tandem spheres with collision effect. J. Vis. 23, 49-59.

ZhAO, M.-H., CHEN, X.-P. \& WANG, Q. 2014 Wetting failure of hydrophilic surfaces promoted by surface roughness. Sci. Rep. 4 (1), 33-5. 